\title{
GEOPHYSICS
}

\section{Regularized full-waveform inversion with automated salt- flooding}

\begin{tabular}{|r|l|}
\hline Journal: & Geophysics \\
\hline Manuscript ID & GEO-2018-0146.R3 \\
\hline Manuscript Type: & Technical Paper \\
\hline Keywords: & full-waveform inversion, Salt, salt dome, subsalt \\
\hline Area of Expertise: & Seismic Inversion \\
\hline \multicolumn{2}{l}{} \\
\end{tabular}

\section{SCHOLARONE ${ }^{\mathrm{m}}$ \\ Manuscripts}




\title{
Regularized full-waveform inversion with automated salt-flooding
}

\author{
Mahesh Kalita $^{1}$, Vladimir Kazei ${ }^{1}$, Yunseok Choi $^{2}$, and Tariq Alkhalifah ${ }^{1}$ \\ ${ }^{1}$ Department of Physical Science and Engineering, \\ King Abdullah University of Science and Technology, \\ Thuwal 23955-6900, Saudi Arabia.
}

E-mail:mahesh.kalita.2@kaust.edu.sa; vladimirkazei@kaust.edu.sa; tariq.alkhalifah@kaust.edu.sa

${ }^{2}$ Formerly, Department of Physical Science and Engineering, King Abdullah University of Science and Technology, Thuwal 23955-6900, Saudi Arabia; Presently, Korea Institute of Geoscience and Mineral Resources, Petroleum and Marine Research Division, Daejeon, South Korea. Email:ychoi@kigam.re.kr (March 20, 2019) Running head: FWI for salt-bodies

ABSTRACT

Full-waveform inversion (FWI) attempts to resolve an ill-posed non-linear optimization problem in order to retrieve the unknown subsurface model parameters from seismic data. Generally, FWI fails to obtain an adequate representation of models with large high-velocity structures over a wide region, like salt bodies and the sediments beneath them, in the absence of low frequencies in the recorded seismic signal, due to non-linearity and non-uniqueness. We alleviate the ill-posedness of FWI associated with datasets affected by salt bodies using model regularization. We split the optimization problem into two parts: first, we minimize the data misfit and the total variation in the
\end{abstract}


model, seeking to achieve an inverted model with sharp interfaces; and second, we minimize sharp velocity drops with depth in the model. Unlike conventional industrial salt flooding, our proposed technique requires minimal human intervention and no information about the top of the salt. Those features are demonstrated on datasets of the BP 2004 and Sigsbee2A models, synthesized from a Ricker wavelet of dominant frequency $5.5 \mathrm{~Hz}$ and minimum frequency $3 \mathrm{~Hz}$. We initiate the inversion process with a simple model in which the velocity increases linearly with depth. The model is well retrieved when the same constant density acoustic code is used to simulate the observed data, which is still one of the most common FWI tests. Moreover, our technique allows us to reconstruct a reasonable depiction of the salt structure from the data synthesized independently with the BP 2004 model with variable density. In the Sigsbee2A model, we manage even to capture some of the fine layering beneath the salt. In addition, we show the versatility of our method on a field dataset from the Gulf of Mexico. 


\section{INTRODUCTION}

Velocity models are very challenging to build from seismic data, especially for complex salt provinces. Various factors, such as data enrichment by strong multiples, poor sampling due to limitations in the acquisition process, and incomplete subsurface illumination caused by velocity complexities in the overburden, contribute to the failure of conventional computational practices. As of now, there remain many and varied pitfalls at every stage of the building process for velocity models of saltaffected datasets. Attempts to identify and then mitigate these challenges often require both geophysical skills and geological interpretation (Farmer et al., 1996; Jones, 2012; McCann et al., 2014; Jones and Davison, 2014). The conventional industrial practice 'migrate-pick-flood' (Dellinger et al., 2017) is subject to the skill level of human professionals, is reiterative by nature, and often exacerbates the already difficult process of velocity model building, thus affecting the quality of the subsurface images. In addition, it impedes the ultimate goal of seismic tomography: replacing human interpretation with computational methods.

Full-waveform inversion (FWI) aims to recover the unknown model parameters from seismic data by minimizing the misfit between observed and modeled data (Lailly, 1983; Tarantola, 1984; Pratt, 1990). Despite continued improvements in computational power over recent years, FWI still requires that model perturbations be evaluated based on a single scattering assumption, known as the Born approximation. As a result, FWI fails to render the non-linear quantitative parameters from the seismic data, given that the initial model is very far from the true subsurface structure. This limitation is aggravated in the presence of a salt body about whose position and shape the initial model most likely has no a priori knowledge, and whose physical properties (e.g. high velocity and low density) are completely distinct from those of its neighborhood. Therefore, the Born assumption breaks down in this scenario, especially in the absence of ultra low frequencies, resulting in a failure 


\footnotetext{
${ }^{1}$ The differences between sedimentary velocities of the true and initial models in Lewis and Vigh (2016) is not zero. The authors prepare the initial model by smoothing and subsequently expanding the true sediment velocity. Although misplaced, the initial model nevertheless has a priori knowledge about the salt body.

${ }^{2}$ The examples considered in Kadu et al. (2017) have a very simple sedimentary velocity, i.e., a linearly increasing which is parametrized by its slope.
} 
sedimentary background model or a priori information about the location of the salt dome. With regard to the flooding part, the inequality constraint term of Esser et al. (2016a) represents a special case of our proposed penalty term.

In this article, we attempt to mitigate the perennial question of seismic industry: can the velocity models of salt and subsalt provinces be retrieved from seismic datasets without human intervention? We propose regularizing FWI by penalizing the velocity drops with depth. To do so, we split the minimization problem into two cascading stages: first, we match the data, preserving the sharp edges of the model; and second, we flood from the top of the salt (without picking), which is a post-processing optimization procedure. This split approach is extremely convenient, because it is easy to implement in existing FWI schemes. Moreover, multiple regularization weights can be efficiently tested in the flooding. We begin the article with a theory section on FWI with model regularization, which retrieves the sharp edges of the salt body and subsequently, floods it. Next, we discuss the gradient direction behavior associated with the flooding process. We demonstrate the versatility of our proposed method on the synthetic datasets of the BP 2004 and Sigsbee2A models in which the lowest available frequency is $3 \mathrm{~Hz}$. We begin the inversion process with a very poor model whose velocity increases linearly with the depth and that contains no information of the salt body. The model is well reconstructed when the same constant density acoustic code is used to simulate the observed data. Moreover, our technique still manages to delineate most of the BP 2004 velocity model from the data synthesized independently with the variable density model. Also, we demonstrate its application on a complicated field dataset (for FWI) from Gulf of Mexico. 
FWI has summoned enormous attention in the past decade, owing to its potential to recover unknown model parameters from seismic data (Pratt et al., 1998; Sirgue and Pratt, 2004; Virieux and Operto, 2009). Despite intrinsic difficulties such as cycle-skipping issues, computational overburden, and so on, the capability of FWI to recover parameters from field datasets has been confirmed by numerous studies, e.g., Vigh et al. (2009); Plessix et al. (2010); Prieux et al. (2013); Warner et al. (2013); Ramos-Martinez et al. (2013); Choi and Alkhalifah (2015); Kalita and Alkhalifah (2017); Routh et al. (2017); Oh et al. (2017). Despite its success, FWI, like many geophysical-estimation problems, is an ill-posed mathematical problem that is operated on seismic field data corrupted by noise and inaccurate experiments. Several studies have used model regularization schemes to alleviate this ill-posedness by reinforcing some geologically plausible characteristics in the inverted model. Total-variation (TV) is one of the most popular regularization techniques in seismic tomography (Anagaw and Sacchi, 2012; Loris and Verhoeven, 2012; Esser et al., 2016b). TV is a nonquadratic regularization method that imposes sharp edges without over-smoothing the reconstructed the model (Rudin et al., 1992). Alternatively, Kazei et al. (2017) applied a regularization based on minimum gradient support (MGS) (Portniaguine and Zhdanov, 1999) and the Sobolev space norm $\left(W_{p}^{1}\right)$ in order to maintain a balance between the sharp and smooth updates in FWI. Here, we implement two regularization terms in the context of a salt-body inversion: one that preserves the edge and another that penalizes for the velocity drop, as discussed in the following section.

\section{Problem statement}

Typically, salt bodies are steeply-dipping complex shaped structures with higher velocity and lower density compared to its sedimentary neighborhood. Therefore, an edge-preserving technique, such 
PROBLEM 2 : FLOODING

$\mathbf{d}_{0} \quad$ : observed data

$$
J_{F W I+T V}(\mathbf{m})=\min _{\mathbf{m}}\left\{\left\|\mathbf{d}_{o}-\mathbf{d}_{m}\right\|_{2}^{2}+\lambda_{T V}\|\mathbf{m}\|_{T V}\right\}
$$

$\mathbf{d}_{m}:$ synthetic data that follows the wave equation

$$
\|\mathbf{m}\|_{T V}=|\nabla \mathbf{m}|=\sqrt{\mathbf{m}_{x}^{2}+\mathbf{m}_{z}^{2}} \text { with }
$$

$$
\nabla \mathbf{m}=\left(\mathbf{m}_{x}, \mathbf{m}_{z}\right)=\left(\frac{\partial \mathbf{m}}{\partial x}, \frac{\partial \mathbf{m}}{\partial z}\right)
$$

$\lambda_{T V}:$ regularization coefficient of TV regularization;

$$
J_{F}(\mathbf{q})=\min _{\mathbf{q}}\left\{\|\mathbf{q}-\mathbf{u}\|_{2}^{2}+\lambda_{F}\|\mathbf{q}\|_{F}\right\}
$$




$$
\begin{aligned}
& \mathbf{u} \quad: \underset{\mathbf{m}}{\operatorname{argmin}} J_{F W I+T V} \\
& \|\mathbf{q}\|_{F}: \text { flooding regularization defined as }
\end{aligned}
$$

$$
\|\mathbf{q}\|_{F}=\frac{1}{2 p}\left\|\left(\left|\mathbf{q}_{z}\right|-\mathbf{q}_{z}\right)^{p}\right\|_{2}^{2} \quad \text { with } \quad p>1 / 2
$$

$\lambda_{F}:$ regularization coefficient of $\|\mathbf{q}\|_{F}$.

The first minimization problem, 'FWI+TV', recovers the velocity field by promoting limited variation regions in the model. Here, $\lambda_{T V}$ is a positive regularization parameter that controls the trade-off between the data misfit term and the amount of interface-preservation in the inversion process. The second optimization problem, flooding, starts with the final output of 'FWI+TV' and penalizes the velocity drop in the model. We call it flooding because the velocity field is smeared along the vertical direction after many iterations. In addition to the advantage of minimal human intervention, flooding is applied anywhere and everywhere since it is governed by the model under consideration instead of the top of the salt. Thus, the high velocity is smeared across any region where there is a drop in the velocity field, rather than only the salt body region. This might have a detrimental effect in the presence of low-velocity zones, especially over the top of the salt. In that case, a minimal human intervention might be required to flood from the top of the salt. However as mentioned later, the $p$ value in equation (4) plays the most significant role in the automated selection of a flooding region in the model.

\section{Gradient evaluation}

In practice, the gradient direction of the data misfit term in FWI is evaluated using the adjoint-state method (Lailly, 1983; Plessix, 2006). The gradient of the TV functional (equation (2)) cannot be 
computed at the origin $\nabla \mathbf{m}=0$. Therefore, we are required to introduce a smoothing parameter, $\epsilon_{T V}$, to the functional as follows:

$$
J_{T V}^{\epsilon}(\mathbf{m})=\sqrt{\mathbf{m}_{x}^{2}+\mathbf{m}_{z}^{2}+\epsilon_{T V}}
$$

Accordingly, its gradient direction is given by

$$
\frac{\delta J_{T V}^{\epsilon}}{\delta \mathbf{m}}=-\nabla \cdot \frac{\nabla \mathbf{m}}{J_{T V}^{\epsilon}}
$$

The role of $\epsilon_{T V}$ in the functional is very well known; more details can be found in Kazei et al. (2017). Next, the gradient computation of the flooding regularization, equation (4), is given by

$$
\frac{\delta\|\mathbf{q}\|_{F}}{\delta \mathbf{q}}= \begin{cases}-(2 p-1) 4^{p} \mathbf{q}_{z}^{2(p-1)} \frac{\partial \mathbf{q}_{z}}{\partial z} & \text { for } \quad \mathbf{q}_{z}<0 \\ 0 & \text { otherwise. }\end{cases}
$$

Equation (7) suggests that the gradient direction of the flooding regularization is primarily governed by the quantity $-\mathbf{q}_{z}^{2(p-1)} \frac{\partial \mathbf{q}_{z}}{\partial z}$. Careful observation of this quantity reveals that the parameter $p>1$ favors the gradient direction towards a region of steeper velocity drops. A value of $1 / 2<p \leq 1$ sets $\mathbf{q}_{z}^{2(1-p)}$ as a denominator in equation (7), thus, producing an impartial gradient, albeit an undesired one in the context of salt flooding. Please refer to the appendix for a detailed derivation of the gradient computation using calculus of variations. 


\section{Gradient preconditioning of flooding regularization}

We consider a dummy 1D velocity profile, given by the sine function of depth, to analyze the behavior of the gradient direction during flooding regularization. The velocity profile (red) and the update direction (negative gradient) (green) are displayed in Figure 1. As expected, the update is zero while the velocity increases with depth. However, when the velocity drops, the non-zero update is not always positive, which reverses the direction. Anticipating this, we update the velocity field only when the update direction is positive, as shown by the black profile in Figure 1.

\section{Role of $p$}

We study the functional behavior of the flooding regularization with respect to $p$ and $\mathbf{q}_{z}$ in Figure 2 .

We see that the functional given by equation (4) is biased towards a steeper velocity drop for large $p$, and is almost insensitive to smaller decreases in velocity. We verify this observation by evaluating the gradient of the velocity profile shown in Figure 3. The velocity profile contains three noticeable low-velocity zones, marked as (1), (2), and (3) according to their ascending order of the steepness. For $p=1$, the update direction responds to even minute changes in the velocity profile. On the other hand, there are two non-zero regions for $p=2$ and only one for $p=4$ in the update direction, confirming that the functional with large $p$ is immune to small-scale changes in the velocity field. Therefore, in the context of our study, we maneuver the update direction towards a region of steeper velocity drops by setting $p$ high enough. A steeper drop, fortunately, occurs just below the top of the salt-body for the numerical problems under our consideration. 

the pseudo-code in Algorithm 1 and 2.

\section{Implementation strategy}

As mentioned above, we decouple the full problem into the two parts: 'FWI+TV' and 'flooding'. Both of them can be solved using any method of the non-linear optimization framework, such as steepest-descent or L-BFGS, etc. We opt for the non-linear conjugate gradient method and provide

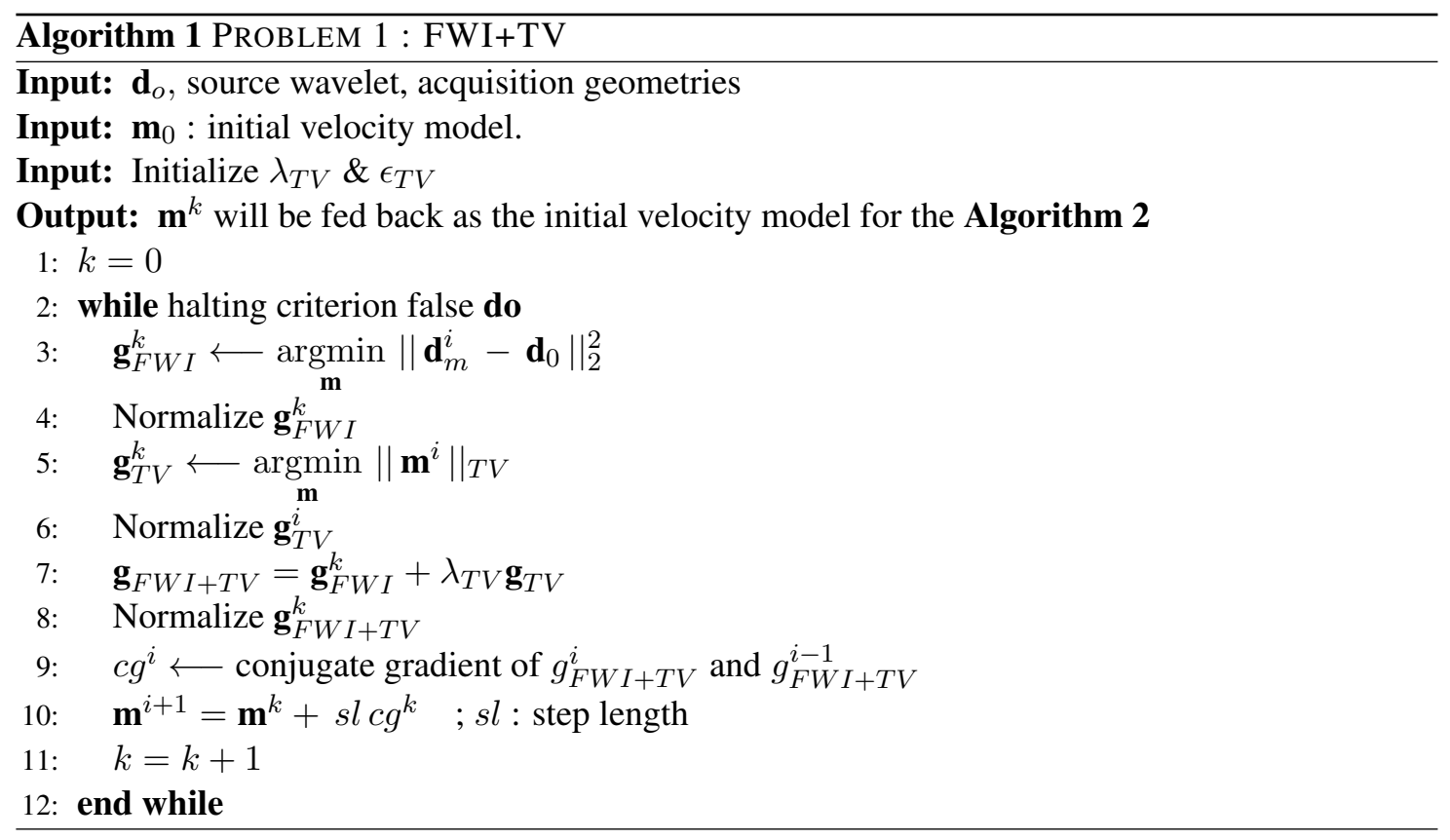

178

In any non-linear regularization problem, the smoothing parameter $\epsilon$ and the regularization coefficient $\lambda$ control the success and convergence rate of the problem. A large $\epsilon$ leads to a smoother solution, which is undesirable since we want to preserve the sharp edges in a model with high resolution. The regularization coefficient $\lambda$ implies the confidence level between the terms involved in the functional. Therefore, an appropriate selection of $\epsilon$ and $\lambda$ is very important for the effective application of model regularization. Here, we set $\epsilon_{T V}$ to be very small, depending on the model parameters. For example, we set $\epsilon_{T V}$ to $0.1 \%$ of $\overline{<\nabla \mathbf{m}, \nabla \mathbf{m}>}$. $^{3}$

${ }^{3} \overline{\langle\mathbf{a}, \mathbf{b}\rangle}$ signifies the dot product of $\mathbf{a}$ and $\mathbf{b}$ divided by the total number of elements in the vector $\mathbf{a}$. 


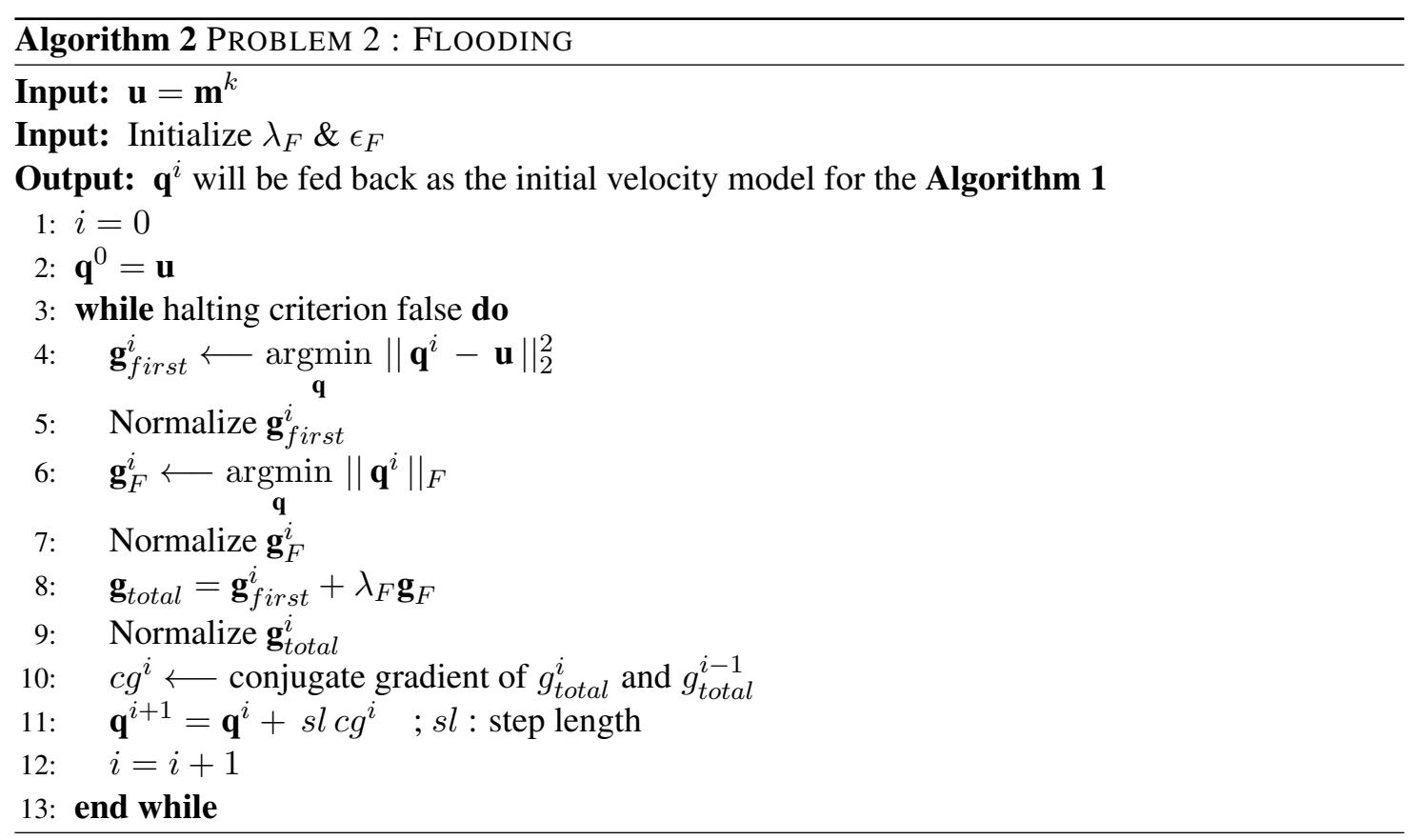

Although several robust selection procedure methods for $\lambda_{T V}$ exist (Solo, 1999; Lin et al., 2010; Guitton, 2012; Lin and Huang, 2014), we apply the very simple selection procedure of assigning a stationary number for each bandwidth of the data. For example, $\lambda_{T V}=0.5$ for the first bandwidth, $\lambda_{T V}=0.3$ for the second bandwidth of data, and so on. Therefore, the data misfit term in 'FWI+TV' gradually dominates the inversion, which produces results that are less biased by the initial values of the regularization parameters. In addition, the final stage of the inversion using the full-bandwidth of the data is purely data driven without any model regularization. In other words, we solve only 'FWI+TV' with $\lambda_{T V}=0$ for the full bandwidth of the data. For the second optimization problem, 'flooding', we assign $p=2$ and $\lambda_{F}=10 * \lambda_{T V}$.

\section{Features of our flooding procedure}

In this section, we shed some light on the contrasting methodologies in this article and of Esser et al. (2016a). In their study, Esser et al. dealt with the constrained optimization problem as a set of 
'hard-constraints', which mathematically can be formulated as:

$$
\begin{aligned}
& J(\mathbf{m})=\min _{\mathbf{m}}\left\{\left\|\mathbf{d}_{o}-\mathbf{d}_{m}\right\|_{2}^{2}\right\}, \text { which is } \\
& \text { subject to } \quad\|m\|_{T V} \leq \tau_{1} \\
& \text { and } \quad\left\|\min \left(0, \mathbf{m}_{z}\right)\right\|_{1} \leq \tau_{2},
\end{aligned}
$$

\footnotetext{
${ }^{4}$ Since the final inverted results should be governed by the data fidelity term more than the model regularization, we believe the both implementations of the model regularization will render the same results, especially from a field dataset.
} 
- In contrast to their formulation, we split the optimization problem into two separate segments: 'FWI+TV' and 'flooding'. We activate the flooding regularization on a more mature and refined velocity model in which the top of the salt region has already been reconstructed, rather than at every iteration of FWI as Esser et al. (2016a) do. This concept of a preconstructed top of the salt before flooding is essentially in line with the industrial strategy, but the lack of assumption about the homogeneous velocity inside the salt dome and the picking make it mathematically more robust.

- Thanks to our split approach, we can efficiently facilitate the trial and error strategy that is necessary for the computation of the regularization weights in the flooding part without any incurring significant computational costs.

\section{EXAMPLES}

\section{BP 2004 velocity model}

We demonstrate an application of the proposed method to the west part of the BP 2004 velocity model (Billette and Brandsberg-Dahl, 2005). As is often done with this model in inversion tests, we adjust the size to half of its original size, but we keep the aspect ratio unaltered for our study. It contains a simple background with a complex, rugose salt body. There are several low-velocity subsalt anomalies and complex overhangs, which attempt to imitate the deep-water prospect areas in the Gulf of Mexico. Figure 4 displays the true synthetic model of our consideration.

The model of consideration has 801 and 216 grid points along the $x$ and $z$ directions, respectively, at equal intervals of $0.020 \mathrm{~km}$. There are 248 sources placed between $x=0.5 \mathrm{~km}$ and $x=15.44 \mathrm{~km}$ at intervals of $0.06 \mathrm{~km}$. All the sources are placed at a depth of $z=0.02 \mathrm{~km}$. The data are recorded by a streamer of $16.0 \mathrm{~km}$ fixed length, which is placed at the same depth as the 
source line for 10 seconds. A Ricker wavelet of $5.5 \mathrm{~Hz}$ dominant frequency and $3 \mathrm{~Hz}$ minimum frequency is used to synthesize the observed data with the free surface boundary condition. Figures 5(a) and 5(b) show the source wavelet and its frequency content, respectively. Also, we display two shot gathers from sources placed at $x=0.5 \mathrm{~km}$ and $x=8.0 \mathrm{~km}$ in Figures 6(a) and 6(b), respectively. We display the frequency content of the data from Figure 16(b) in Figure 16(c).

We kick off the inversion process with the velocity model displayed in Figure 7. The velocity increases linearly with the depth from the water bottom. More importantly, the model contains no prior knowledge of the salt's position or velocity. We follow the multiscale approach described in Bunks et al. (1995), starting the inversion at the lower frequency bandwidth and progressively including the higher frequencies. We consider frequencies less than $3.5 \mathrm{~Hz}$, followed by $4 \mathrm{~Hz}, 6 \mathrm{~Hz}$ and full bandwidth of the data in the inversion scheme. Figure 8 shows the final inverted model of the first optimization problem, i.e., 'FWI+TV'. It manages to retrieve the top of the salt, but fails to retrieve the vicinity of the salt as pointed out by the encircled region in Figure 8 . We subsequently subject the final inverted model to flooding. A couple snapshots of the flooding process are displayed in Figure 9. The flooding manages to wipe out the sudden drop in velocity below the top of the salt. However, it pays the price of the flooding at other regions as well, e.g., the areas designated by blue circles in Figure 9(b). We feed the final output of the flooding process back into the 'FWI+TV' scheme. The data misfit term rescues the region from over-flooding, and yields a model close to the true one, as displayed in Figure 10. Next, this velocity model is again subjected to the 'flooding' optimization problem. As shown in Figure 11, this procedure removes velocity drops, especially beneath the salt; thus, it damages the already reconstructed subsalt zone. However, a subsequent 'FWI+TV' retrieves back those velocity drops as shown in Figure 12. As expected, the inversion starts by building the top of the salt correctly, then slowly propagates further throughout its vicinity. Once the salt body has been retrieved, it reattempts to reconstruct the subsalt region. Since the 
velocity models shown in Figure 10 and the bottom panel of Figure 12 look similar, we do not stimulate further 'flooding'. Instead, we move on to 'FWI+TV' with $\lambda_{T V}=0$, i.e., a conventional FWI, to obtain the final inverted model (Figure 13). As indicated by the blue circle in Figure 13, our proposed method is able to retrieve even small intrusions inside the salt dome, which are often referred to dirty salt. ${ }^{5}$ A comparison between Figures 13 and 14 confirms that the method proposed in this article reconstructs the salt and subsalt regions much better than FWI alone.

Next, we perform the optimization problem without the inverse crime. We incorporate a variable density (Figure 15) in the simulation for the observed data using a program named sfawefd $2 d$ from the open-source software package named Madagascar (Fomel et al., 2013). We retain the same source wavelet and the acquisition geometry in order to prepare the dataset. Figures 16(a) and 16(b) display two shot gathers from sources placed at $x=0.5 \mathrm{~km}$ and $x=8 \mathrm{~km}$, respectively. We display the average frequency spectrum of the data from Figure 16(b) in Figure 16(c). However, we replace the $L^{2}$ norm of data misfit with the normalized cross-correlation objective function (Routh et al., 2011; Choi and Alkhalifah, 2012). By performing our two-problem strategy with constant density acoustic wave-equation as above, we obtain the inverted model (Figure 17). As expected, inversion cannot reconstruct the model as good as in the constant density data case (Figure 13). Nevertheless, we managed to delineate most of the salt velocity model. Thus, it demonstrates the algorithm's potential adaptability to a realistic setting where the wave propagation is much more complex.

\section{Sigsbee2A}

We consider the Sigsbee2A model (Paffenholz et al., 2002) to demonstrate the versatility of our proposed method. However, we reduce the thickness of the water column of the original model by

\footnotetext{
${ }^{5}$ In general, industrial manual flooding assumes that the velocity is homogeneous inside the salt dome; hence, it fails to retrieve the dirty salts.
} 
$1.5 \mathrm{~km}$. Figure 18 shows the true model of our consideration. Besides the complex salt structure with its variable size and shape, this model contains a sedimentary sequence broken up by a number of normal and thrust faults. The source wavelet shown in Figure 5(a) is used to prepare the observed data, which is recorded in a $16 \mathrm{~km}$ long streamer, starting from $0.16 \mathrm{~km}$ at the near offset, placed at a depth of $0.016 \mathrm{~km}$. Unlike the previous example, we set the streamer length to vary with the position of the sources in order to emulate the typical marine acquisition geometry. Figure 19 confirms the poor acquisition coverage towards the left part of the model. We distribute 360 sources between $x=0.480 \mathrm{~km}$ to $x=23.52 \mathrm{~km}$ at the surface, $z=0.016 \mathrm{~km}$. Figures 20 (a) and 20(b) show two shot gathers from sources placed at $x=3.04 \mathrm{~km}$ and $x=23.52 \mathrm{~km}$, respectively. Figure 20(c) displays the average frequency content of the shot-gather shown in Figure 20(b). We prepare the initial model (Figure 21(a)) by increasing the velocity linearly from the water bottom.

The final inverted model, displayed in Figure 21(b), shows that we retrieved the salt-body very well. The inversion tries to render the smooth version of the subsalt, which looks very promising for the imaging purpose. In order to retrieve the reflectors in high resolution, we run basic FWI on the model displayed in Figure 21(b) by preconditioning the gradient to zero whenever the velocity exceeds $4.2 \mathrm{~km} / \mathrm{s}$. Figure 21(c) displays the high resolution result. An enlarged area from Figure 21(c) is shown in Figure 22 for comparison. By taking into account the innate limitations of FWI, such as poor subsurface illumination, and limited acquisition aperture, we are able to retrieve most of the model.

\section{Field data application}

This example contains a vintage 2D marine seismic dataset acquired in a region of Gulf of Mexico (GOM) during the decade of 1990. The data were recorded using a short streamer of length $4.8 \mathrm{~km}$, 
containing 180 receivers with the near offset of $0.1 \mathrm{~km}$. The lowest usable frequency in the dataset is around $7.0 \mathrm{~Hz} .{ }^{6}$ We consider the model to be $21.6 \mathrm{~km}$ long and $4.5 \mathrm{~km}$ deep. Figure 23 displays one shot gather from the source placed at $x=11.0 \mathrm{~km}$ and its frequency content.

We start the inversion process with a model shown in Figure 24. We keep the water layer velocity unchanged throughout the inversion scheme. As expected, 'FWI+TV' scheme retrieves the position of the top of the salt (Figure 25(a)). Next, this model is subjected to the 'flooding' optimization problem. It removes the velocity drops beneath the recovered top of the salt as shown in Figure 25(b). A couple of additional snapshots of inversion procedure are shown in Figure 25 to portray the evolution of the model. Finally, we retrieve the inverted model as shown in Figure 26. We manage to recover the top and bottom boundaries of the salt dome. Unlike the previous synthetic examples of consideration, in particular of BP 2004 model (Figure 12), this seismic dataset did not manage to wipe out the undue flooding as shown by the blue arrows in Figure 26 because of its innate limitations such as very limited offset (only $4.8 \mathrm{~km}$ ) and the absence of low frequencies $(7.0 \mathrm{~Hz})$. Low wavenumber components in the update are needed to correct the flooding influence automatically.

We validate the inversion result by the following $\mathrm{QC}$ measures:

- Data comparison: We interleave observed and predicted seismic data from a source located at $x=11.0 \mathrm{~km}$. Figure 27(a) shows the matching of data from the initial model and Figure 27(b) from the final model. The inverted model produces a data set which resembles the observed recording better than the initial model does.

- Migration image comparison: As shown in Figure 28, we observe improvements in the reverse time migration (RTM) image using the final inverted model. The initial velocity

\footnotetext{
${ }^{6}$ In general, this type of acquisition geometry and the available frequency content of the dataset are not best suited for successful FWI given a poor starting model.
} 
model fails to delineate the bottom of the salt region as shown in Figure 28(a), whereas the final velocity model clearly accentuates the shape of the salt-dome as shown in Figure 28(b).

- Angle domain common image gather (ADCIG) comparison: The erroneous velocities produce non-flat events in ADCIGs. We have computed ADCIGs at an interval of $0.7 \mathrm{~km}$, starting from $6.0 \mathrm{~km}$ to $17.0 \mathrm{~km}$ in Figure 29. It shows some of the events, as pointed out by the arrows, are flatter with the inverted model compared to those using the initial model.

With this challenging field data example for FWI, we managed to highlight some of the limitations of this flooding based FWI, which is synonymous with the limitations we expect from FWI. A closer look at the inverted model suggests that the bottom of the salt might have been captured. However, the ability of the inversion to correct the velocity below the salt hinged on the availability of long model wavenumber updates capable of reversing the flooding. In this dataset, the lack of low frequencies and the limited available offsets contributed to this limitation. In fact, those limitations can be explained with the help of synthetic examples presented in this article:

- In the case of BP-2004 model, we have $16.0 \mathrm{~km}$ long receiver line which in hindsight proves to be long enough for recovering the susbsalt region as shown in Figure 13. On the other hand, the $16 \mathrm{~km}$ long streamer is not good enough for the Sigsbee 2A model. For instance, we could not remove the over-flooding from the subsalt region as indicated by the ellipsees in Figure 21. Under such a thick salt, the data, in spite of the the long streamer, could not illuminate the subsalt with enough low model wavenumber energy to undue the flooding. Thus, the correction has had only high wavenumber content and was focused to capture the bottom of the Salt. Unfortunately, the field dataset of consideration has only $4.8 \mathrm{~km}$ long spread.

- The observed data for the synthetic examples compared to the GOM field data are richer 
in low-frequency bandwidth. Therefore, they enjoy deeper penetration in the model, and, hence offer more in the retrieval process of subsalt region compared to those of the field data example.

- We have shown the versatility of our proposed method on a seismic datset governed by different physics than the underlying constant density acoustic wave equation of our inversion scheme (Figure 17). To make full use of the limited GOM field dataset, however, we cannot overrule the application of better suitable physics such as including elastic/anisotropic behavior of wave propagation to the inversion machinery. In addition, the utilization of multiscattered events, which are prevalent in any salt-flank affected dataset, might expedite the recovery process of residual features in the inverted model (Alkhalifah and $\mathrm{Wu}, 2016$ ). A detailed investigation is required towards that goal.

\section{DISCUSSIONS}

Instead of picking the top of the salt body for flooding, in the proposed method we cast the problem into a better, automated mathematical framework that involves a minimization problem with model regularization. We note that our TV and flooding regularizations also require some user intervention to follow the very simple workflow used to select the parameters, which depend on the model properties, though this intervention is negligible compared to the amount involved in industrial flooding. We still manage to retrieve most, if not all, of the model. Alternate efficient algorithms like the split-Bregman method could reduce the dependency of the model on the smoothing parameter and accelerate its convergence to the true solution (Huang et al., 2008; Goldstein and Osher, 2009; Lin and Huang, 2014). However, we stick with the basic implementations of model regularization since the data misfit term, sooner or later, does take over the inversion scheme, which makes the 
results less biased by the initial values of the regularization parameters. It is worth mentioning that although TV regularization helps FWI reconstruct missing wavenumbers in the model, it has its own limitations (Alkhalifah et al., 2018; Kazei and Alkhalifah, 2018).

The large computational cost of the first problem, 'FWI+TV', which involves more than 1000 non-linear conjugate gradient iterations, appear to be the bottleneck of this method, especially for large 3D problems. Unlike the 'FWI+TV' problem, the flooding process requires negligible computational time because it does not require wavefield extrapolation. Other methods, such as adaptive waveform inversion (Warner and Guasch, 2016), FWI with approximate Gauss-Newton methods (Hou and Symes, 2016), multiscale FWI using a flux-corrected transport technique (Kalita and Alkhalifah, 2018, 2019), or modification in the objective function like the optimal transport distance (Métivier et al., 2016; Yang and Engquist, 2017; Sun and Alkhalifah, 2018) or scattering-angle based preconditioning of FWI gradients (Alkhalifah, 2014; Kazei et al., 2016) have the potential to expedite convergence in the context of inverting for salt-body and remain a topic for future study.

\section{CONCLUSIONS}

We have attempted to address the perennial question on feasibility of 'automated' velocity model building process, especially for a salt-body-affected seismic dataset. In that regard, we have developed FWI with model regularization. We split the minimization problem into two parts, namely 'FWI+TV' and 'flooding'. The 'FWI+TV' promotes the condensed variations in the model obtained from the data misfit function. The 'flooding' smears the high velocity vertically in a controlled manner wherever there is a drop. Any undesired flooding will be eradicated by the data misfit term in the subsequent 'FWI+TV' stage of our cascading strategy. It is mathematically more robust and less cumbersome than the standard 'migrate-pick-flood' method. We have shown the application 


$$
\frac{\delta J}{\delta f}=\frac{\partial L}{\partial f}-\frac{d}{d x} \frac{\partial L}{\partial f^{\prime}} .
$$




$$
\frac{\delta J}{\delta f}=-\frac{d}{d x} \frac{\partial L}{\partial f^{\prime}}
$$

396

\section{TV}

397 We consider the TV functional, defined as

$$
\begin{aligned}
J_{T V} & =\sqrt{\mathbf{m}_{x}^{2}+\mathbf{m}_{z}^{2}} ; \quad \mathbf{m}_{x}=\frac{\partial \mathbf{m}}{\partial x} ; \quad \mathbf{m}_{z}=\frac{\partial \mathbf{m}}{\partial z} \\
& =\int \mathbf{d x} \sqrt{m_{x}^{2}+m_{z}^{2}}
\end{aligned}
$$

Therefore, the gradient of (14) with respect to $m$ (using equation (13)) is given by

$$
\frac{\delta J_{T V}}{\delta m}=-\frac{\partial}{\partial x} \frac{\partial \sqrt{m_{x}^{2}+m_{z}^{2}}}{\partial m_{x}}-\frac{\partial}{\partial z} \frac{\partial \sqrt{m_{x}^{2}+m_{z}^{2}}}{\partial m_{z}}
$$

Since $J_{T V}$ is not differentiable at the origin $\nabla \mathbf{m}=0$, we need to add one parameter, $\epsilon_{T V}$,

$$
J_{T V}^{\epsilon}=\sqrt{\mathbf{m}_{x}^{2}+\mathbf{m}_{z}^{2}+\epsilon_{T V}}
$$

Therefore,

$$
\begin{aligned}
\frac{\delta J_{T V}^{\epsilon}}{\delta m} & =-\frac{\partial}{\partial x}\left(\frac{m_{x}}{J_{T V}^{\epsilon}}\right)-\frac{\partial}{\partial z}\left(\frac{m_{z}}{J_{T V}^{\epsilon}}\right) \\
& =-\nabla \cdot \frac{\nabla m}{J_{T V}^{\epsilon}}
\end{aligned}
$$




\section{Flooding regularization}

We consider the following functional that penalizes the velocity drop with increasing $z$ :

$$
\begin{aligned}
\|\mathbf{m}\|_{F} & =\frac{1}{2 p}\left\|\left(\left|\mathbf{m}_{z}\right|-\mathbf{m}_{z}\right)^{p}\right\|_{2}^{2} ; p>1 / 2 \\
& =\frac{1}{2 p} \int \mathbf{d x}\left(\left|m_{z}\right|-m_{z}\right)^{2 p}
\end{aligned}
$$

$$
\text { Suppose, } f=\left|m_{z}\right|-m_{z} ; \quad \Longrightarrow \frac{\partial f}{\partial m_{z}}=\operatorname{sgn}\left(m_{z}\right)-1 \text {. }
$$

Now, the gradient becomes:

$$
\begin{aligned}
\frac{\delta\|\mathbf{m}\|_{F}}{\delta m} & =-\frac{1}{2 p} \frac{\partial}{\partial z} \frac{\partial f^{2 p}}{\partial m_{z}} \\
& =-\frac{1}{2 p} \frac{\partial}{\partial z}\left[2 p f^{2 p-1}\left\{\frac{\partial\left|m_{z}\right|}{\partial m_{z}}-1\right\}\right] \\
& =-\frac{\partial}{\partial z}\left[f^{2 p-1}\left\{\frac{\partial\left|m_{z}\right|}{\partial m_{z}}-1\right\}\right] \\
& =-\left\{\frac{\partial\left|m_{z}\right|}{\partial m_{z}}-1\right\} \frac{\partial f^{2 p-1}}{\partial z}-f^{2 p-1} \frac{\partial}{\partial z}\left\{\frac{\partial\left|m_{z}\right|}{\partial m_{z}}-1\right\} \\
& =-\left\{\operatorname{sgn}\left(m_{z}\right)-1\right\}(2 p-1) f^{2 p-2}\left\{\operatorname{sgn}\left(m_{z}\right)-1\right\} \frac{\partial m_{z}}{\partial z}-f^{2 p-1} \frac{\partial}{\partial z} \operatorname{sgn}\left(m_{z}\right) \\
& =-(2 p-1) f^{2 p-2}\left\{\operatorname{sgn}\left(m_{z}\right)-1\right\}^{2} \frac{\partial m_{z}}{\partial z}-f^{2 p-1} \frac{\partial}{\partial z} \operatorname{sgn}\left(m_{z}\right) \\
\Longrightarrow \frac{\delta\|\mathbf{m}\|_{F}}{\delta m} & =\left\{\begin{array}{lc}
-(2 p-1) 4^{p} m_{z}^{2(p-1)} \frac{\partial m_{z}}{\partial z} & \text { for } m_{z}<0 \\
0 & \text { otherwise. }
\end{array}\right.
\end{aligned}
$$
steeper velocity drops compared to the rest of the model. 


\section{REFERENCES}

Alkhalifah, T., 2014, Scattering-angle based filtering of the waveform inversion gradients: Geophysical Journal International, 200, no. 1, 363-373.

Alkhalifah, T., B. B. Sun, and Z. Wu, 2018, Full model wavenumber inversion: Identifying sources of information for the elusive middle model wavenumbers: Geophysics, 83, no. 6, R597-R610.

Alkhalifah, T., and Z. Wu, 2016, Multiscattering inversion for low-model wavenumbers: Geophysics, 81, no. 6, R417-R428.

Anagaw, A. Y., and M. D. Sacchi, 2012, Edge-preserving seismic imaging using the total variation method: Journal of Geophysics and Engineering, 9, no. 2, 138.

Billette, F., and S. Brandsberg-Dahl, 2005, The 2004 BP velocity benchmark: Presented at the 67th EAGE Conference \& Exhibition.

Bunks, C., F. M. Saleck, S. Zaleski, and G. Chavent, 1995, Multiscale seismic waveform inversion: Geophysics, 60, no. 5, 1457-1473.

Choi, Y., and T. Alkhalifah, 2012, Application of multi-source waveform inversion to marine streamer data using the global correlation norm: Geophysical Prospecting, 60, no. 4, 748-758.

— 2015 , Unwrapped phase inversion with an exponential damping: Geophysics, 80, no. 5, R251-R264.

Dellinger, J., A. J. Brenders, J. Sandschaper, C. Regone, J. Etgen, I. Ahmed, and K. Lee, 2017, The garden banks model experience: The Leading Edge, 36, no. 2, 151-158.

Esser, E., L. Guasch, F. J. Herrmann, and M. Warner, 2016a, Constrained waveform inversion for automatic salt flooding: The Leading Edge, 35, no. 3, 235-239.

Esser, E., L. Guasch, T. van Leeuwen, A. Y. Aravkin, and F. J. Herrmann, 2016b, Total-variation regularization strategies in full-waveform inversion: arXiv preprint arXiv:1608.06159.

Farmer, P., D. Miller, A. Pieprzak, J. Rutledge, and R. Woods, 1996, Exploring the subsalt: Oilfield 
Review, 8, no. 1, 50 .

Fomel, S., P. Sava, I. Vlad, Y. Liu, and V. Bashkardin, 2013, Madagascar: Open-source software project for multidimensional data analysis and reproducible computational experiments: Journal of Open Research Software, 1, no. 1.

Goldstein, T., and S. Osher, 2009, The split Bregman method for L1-regularized problems: SIAM journal on imaging sciences, 2, no. 2, 323-343.

Guitton, A., 2012, Blocky regularization schemes for full-waveform inversion: Geophysical Prospecting, 60, no. 5, 870-884.

Hou, J., and W. Symes, 2016, Approximate Gauss-Newton iteration for full-waveform inversion, in SEG Technical Program Expanded Abstracts 2016: Society of Exploration Geophysicists, 1163 1168.

Huang, Y., M. K. Ng, and Y.-W. Wen, 2008, A fast total variation minimization method for image restoration: Multiscale Modeling \& Simulation, 7, no. 2, 774-795.

Jones, I. F., 2012, Tutorial: Incorporating near-surface velocity anomalies in pre-stack depth migration models: First Break, 30, no. 3, 47-58.

Jones, I. F., and I. Davison, 2014, Seismic imaging in and around salt bodies: Interpretation, 2, no. 4, SL1-SL20.

Kadu, A., T. van Leeuwen, and W. A. Mulder, 2017, Salt reconstruction in full-waveform inversion with a parametric level-set method: IEEE Transactions on Computational Imaging, 3, no. 2, $305-$ 315.

Kalita, M., and T. Alkhalifah, 2017, Efficient full waveform inversion using the excitation representation of the source wavefield: Geophysical Journal International.

_ 2018, Multiscale full-waveform inversion using flux corrected transport, in SEG Technical Program Expanded Abstracts 2018: Society of Exploration Geophysicists, 1153-1157. 
— 2019, Flux-corrected transport for full-waveform inversion: Geophysical Journal International.

Kazei, V., and T. Alkhalifah, 2018, Time-lapse waveform inversion regularized by spectral constraints and sobolev space norm, in SEG Technical Program Expanded Abstracts 2018: Society of Exploration Geophysicists, 5487-5491.

Kazei, V., M. Kalita, and T. Alkhalifah, 2017, Salt-body inversion with minimum gradient support and Sobolev space norm regularizations: Presented at the 79th EAGE Conference and Exhibition 2017.

Kazei, V., E. Tessmer, and T. Alkhalifah, 2016, Scattering angle-based filtering via extension in velocity: SEG Technical Program Expanded Abstracts 2016, Society of Exploration Geophysicists, $1157-1162$.

Lailly, P., 1983, The seismic inverse problem as a sequence of before stack migrations: Conference on inverse scattering: theory and application, Society for Industrial and Applied Mathematics, Philadelphia, PA, 206-220.

Lewis, W., B. Starr, and D. Vigh, 2012, A level set approach to salt geometry inversion in fullwaveform inversion, in SEG Technical Program Expanded Abstracts 2012: Society of Exploration Geophysicists, $1-5$.

Lewis, W., and D. Vigh, 2016, 3D salt geometry inversion in full-waveform inversion using a levelset method, in SEG Technical Program Expanded Abstracts 2016: Society of Exploration Geophysicists, $1221-1226$.

Lin, Y., and L. Huang, 2014, Acoustic-and elastic-waveform inversion using a modified totalvariation regularization scheme: Geophysical Journal International, 200, no. 1, 489-502.

Lin, Y., B. Wohlberg, and H. Guo, 2010, UPRE method for total variation parameter selection: Signal Processing, 90, no. 8, 2546-2551. 
Loris, I., and C. Verhoeven, 2012, Iterative algorithms for total variation-like reconstructions in seismic tomography: GEM-International Journal on Geomathematics, 3, no. 2, 179-208.

McCann, D., A. Comas, G. Martin, A. McGrail, and J. Leveille, 2014, Seismic data processing empowers interpretation: A new methodology serves to mesh processing and interpretation: Harts $\mathrm{E} \& \mathrm{P}$.

Métivier, L., R. Brossier, Q. Mérigot, E. Oudet, and J. Virieux, 2016, Measuring the misfit between seismograms using an optimal transport distance: application to full waveform inversion: Geophysical Supplements to the Monthly Notices of the Royal Astronomical Society, 205, no. 1, $345-377$.

Oh, J.-W., M. Kalita, and T. Alkhalifah, 2017, 3D elastic full waveform inversion using p-wave excitation amplitude: Application to OBC field data: Geophysics, 83, no. 2, 1-87.

Ovcharenko, O., V. Kazei, D. Peter, and T. Alkhalifah, 2018a, Variance-based model interpolation for improved full-waveform inversion in the presence of salt bodies: Geophysics, 83, no. 5, 1-60.

Ovcharenko, O., V. Kazei, D. Peter, X. Zhang, and T. Alkhalifah, 2018b, Low-frequency data extrapolation using a feed-forward ann: Presented at the 80th EAGE Conference and Exhibition 2018.

Paffenholz, J., J. Stefani, B. McLain, and K. Bishop, 2002, Sigsbee 2a synthetic subsalt datasetimage quality as function of migration algorithm and velocity model error: 64th annual international meeting, european association of geoscience engineers: Presented at the Extended $\mathrm{Ab}-$ stracts B.

Plessix, R.-E., 2006, A review of the adjoint-state method for computing the gradient of a functional with geophysical applications: Geophysical Journal International, 167, no. 2, 495-503.

Plessix, R.-E., G. Baeten, J. W. de Maag, M. Klaassen, Z. Rujie, and T. Zhifei, 2010, Application of acoustic full waveform inversion to a low-frequency large-offset land data set, in SEG Technical 
Program Expanded Abstracts 2010: Society of Exploration Geophysicists, 930-934.

Portniaguine, O., and M. S. Zhdanov, 1999, Focusing geophysical inversion images: Geophysics, 64, no. 3, 874-887.

Pratt, R. G., 1990, Inverse theory applied to multi-source cross-hole tomography.: Geophysical Prospecting, 38, no. 3, 311-329.

Pratt, R. G., C. Shin, and G. Hick, 1998, Gauss-Newton and full Newton methods in frequencyspace seismic waveform inversion: Geophysical Journal International, 133, no. 2, 341-362.

Prieux, V., R. Brossier, S. Operto, and J. Virieux, 2013, Multiparameter full waveform inversion of multicomponent ocean-bottom-cable data from the Valhall field. Part 1: Imaging compressional wave speed, density and attenuation: Geophysical Journal International, 194, no. 3, 1640-1664.

Ramos-Martinez, J., K. Zou, S. Kelly, and B. Tsimelzon, 2013, Reflection FWI from fully deghosted towed-streamer data: A field data example, in SEG Technical Program Expanded Abstracts 2013: Society of Exploration Geophysicists, 887-891.

Routh, P., J. Krebs, S. Lazaratos, A. Baumstein, S. Lee, Y. H. Cha, I. Chikichev, N. Downey, D. Hinkley, and J. Anderson, 2011, Encoded simultaneous source full-wavefield inversion for spectrally shaped marine streamer data, in SEG Technical Program Expanded Abstracts 2011: Society of Exploration Geophysicists, 2433-2438.

Routh, P., R. Neelamani, R. Lu, S. Lazaratos, H. Braaksma, S. Hughes, R. Saltzer, J. Stewart, K. Naidu, H. Averill, et al., 2017, Impact of high-resolution FWI in the western Black Sea: Revealing overburden and reservoir complexity: The Leading Edge, 36, no. 1, 60-66.

Rudin, L. I., S. Osher, and E. Fatemi, 1992, Nonlinear total variation based noise removal algorithms: Physica D: Nonlinear Phenomena, 60, no. 1-4, 259-268.

Shen, X., I. Ahmed, A. Brenders, J. Dellinger, J. Etgen, and S. Michell, 2018, Full-waveform inversion: The next leap forward in subsalt imaging: The Leading Edge, 37, no. 1, 67b1-67b6. 
Sirgue, L., and R. G. Pratt, 2004, Efficient waveform inversion and imaging: A strategy for selecting temporal frequencies: Geophysics, 69, no. 1, 231-248.

Solo, V., 1999, Selection of regularisation parameters for total variation denoising: Acoustics, Speech, and Signal Processing, 1999. Proceedings., 1999 IEEE International Conference on, IEEE, 1653-1655.

Sun, B., and T. Alkhalifah, 2018, The application of an optimal transport to a preconditioned data matching function for robust waveform inversion, in SEG Technical Program Expanded Abstracts 2018: Society of Exploration Geophysicists, 5168-5172.

Tarantola, A., 1984, Inversion of seismic reflection data in the acoustic approximation: Geophysics, 49, no. 8, 1259-1266.

Vigh, D., W. Starr, and K. K. Dingwall, 2009, 3D prestack time domain full waveform inversion: Presented at the 71st EAGE Conference and Exhibition incorporating SPE EUROPEC 2009.

Virieux, J., and S. Operto, 2009, An overview of full-waveform inversion in exploration geophysics: Geophysics, 74, no. 6, WCC1-WCC26.

Warner, M., and L. Guasch, 2016, Adaptive waveform inversion: Theory: Geophysics.

Warner, M., A. Ratcliffe, T. Nangoo, J. Morgan, A. Umpleby, N. Shah, V. Vinje, I. Štekl, L. Guasch, C. Win, et al., 2013, Anisotropic 3D full-waveform inversion: Geophysics, 78, no. 2, R59-R80.

Yang, Y., and B. Engquist, 2017, Analysis of optimal transport and related misfit functions in FWI: Geophysics, 83, no. 1, 1-25. 


\section{LIST OF FIGURES}

1 A dummy velocity profile (red); its update direction (green) is not free from undesired directions; the black line shows the controlled update.

2 Behavior of the functional $\|\mathbf{q}\|_{F}$ with $p$. At large $p$, the functional is biased towards steeper velocity drops compared to smaller ones.

3 A dummy velocity profile (black) and its update directions with $p=\{1,2,4\}$. The large $p$ makes the update direction immune to small-scale changes in velocity.

4 The west part of the BP 2004 velocity model.

5 (a) The Ricker wavelet and (b) its frequency content in order to simulate the observed dataset. The lowest available frequency in the wavelet is $3.0 \mathrm{~Hz}$.

6 Shot records from the sources placed at (a) $x=0.5 \mathrm{~km}$ and (b) $x=8.0 \mathrm{~km}$, respectively; (c) average spectrum of (b).

7 Initial model to invert for the BP 2004 velocity.

8 Results of the first optimization problem ('FWI + TV'). The blue circle indicates the region where FWI starts failing to update the velocity in the right direction.

9 Snapshots from the second optimization problem ('flooding'). The flooding procedure succeeded in wiping out the wrong velocity of the reconstructed model of 'FWI+TV' (Figure 8). However, we observe undue flooding in some regions, which is indicated by the ellipses in the bottom panel.

10 Output of the 'FWI+TV' scheme with the input velocity from the bottom panel of Figure 9. 'FWI+TV' removed the residuals of undue flooding, and recovered most, including the subsalt, regions of the model.

11 Result of the 'flooding' procedure shown in Figure 10 velocity.

12 (From top to bottom) Evolution of the velocity model using 'FWI+TV'. We decrease the 
regularization coefficient of TV with higher frequency bandwidths.

13 Final reconstructed model of 'FWI+TV' with $\lambda_{T V}=0$ for the full bandwidth of the dataset. We retrieved not only the salt and subsalt regions, but also the dirty salt as indicated by the blue circle.

14 Reconstructed model with no regularization, i.e., conventional FWI with multi-scale approach (Bunks et al., 1995). Compare with Figure 13.

15 Variable density of the BP 2004 model. The density of the salt body is lower than that of the sedimentary neighborhood.

16 Simulated observed data using acoustic wave equation with variable density. The shot gathers for the sources placed at (a) $x=0.5 \mathrm{~km}$ and (b) $x=8.0 \mathrm{~km}$, respectively; (c) average frequency spectrum of data shown in (b). Compare with Figure 6.

17 Final velocity model reconstructed from the observed dataset, displayed in Figure 16.

18 Sigsbee2A velocity model (Paffenholz et al., 2002).

19 Available streamer length for each shot $($ maximum $=16.0 \mathrm{~km}$, minimum $=0.8 \mathrm{~km})$. The acquisition coverage towards the left part of the model is very poor.

20 Shot gathers from the sources placed at (a) $x=2.9 \mathrm{~km}$ and (b) $x=23.0 \mathrm{~km}$; (c) average frequency spectrum of the data shown in (b).

21 (a) Initial model, (b) inverted model, and (c) final inverted model using Figure 21(b) as the initial model, with the gradient preconditioned to zero whenever the velocity is larger than 4.2 $\mathrm{km} / \mathrm{s}$. The inversion process could not remove the unwanted over-flooding from the subsalt region as indicated by the ellipses.

22 Enlarged sections of the (a) true, (b) initial, (c) intermediate (Figure 21(b)), and (d) final inverted (Figure 21(c)) models.

23 (a) A shot gather from the source placed at $x=11.0 \mathrm{~km}$ and (b) its frequency content. 
There is no useful signal below $7 \mathrm{~Hz}$.

24 Initial velocity model to invert available GOM dataset.

25 Evolution of the velocity model through our proposed method: the left panel shows the output of 'FWI+TV' scheme, which are subjected to 'flooding' procedure to obtain the right panel. For 'flooding', we assign $p=4$.

26 Final inverted model using our proposed methodology. The blue arrows indicate the unexpected over flooding residual.

27 Shot gather displaying interleaved predicted and observed data using the (a) initial velocity and (b) final velocity model. We interleave 20 traces starting form the predicted followed by observed data. The blue ellipses confirm that the final inverted velocity model predicts a dataset that match the observed ones better than the initial model does.

28 RTM images using (a) initial velocity and (b) final inverted model. We notice clear boundaries of the salt-dome using the final inverted model, whereas erroneous initial model cannot image those.

29 ADCIGs $\left(0^{\circ}-30^{\circ}\right)$ at an interval of $0.7 \mathrm{~km}$, starting from $x=6.0 \mathrm{~km}$ to $x=17.0 \mathrm{~km}$ using (a) initial and (b) final velocity models. We notice events are flatter using the final model as indicated by the arrows. 


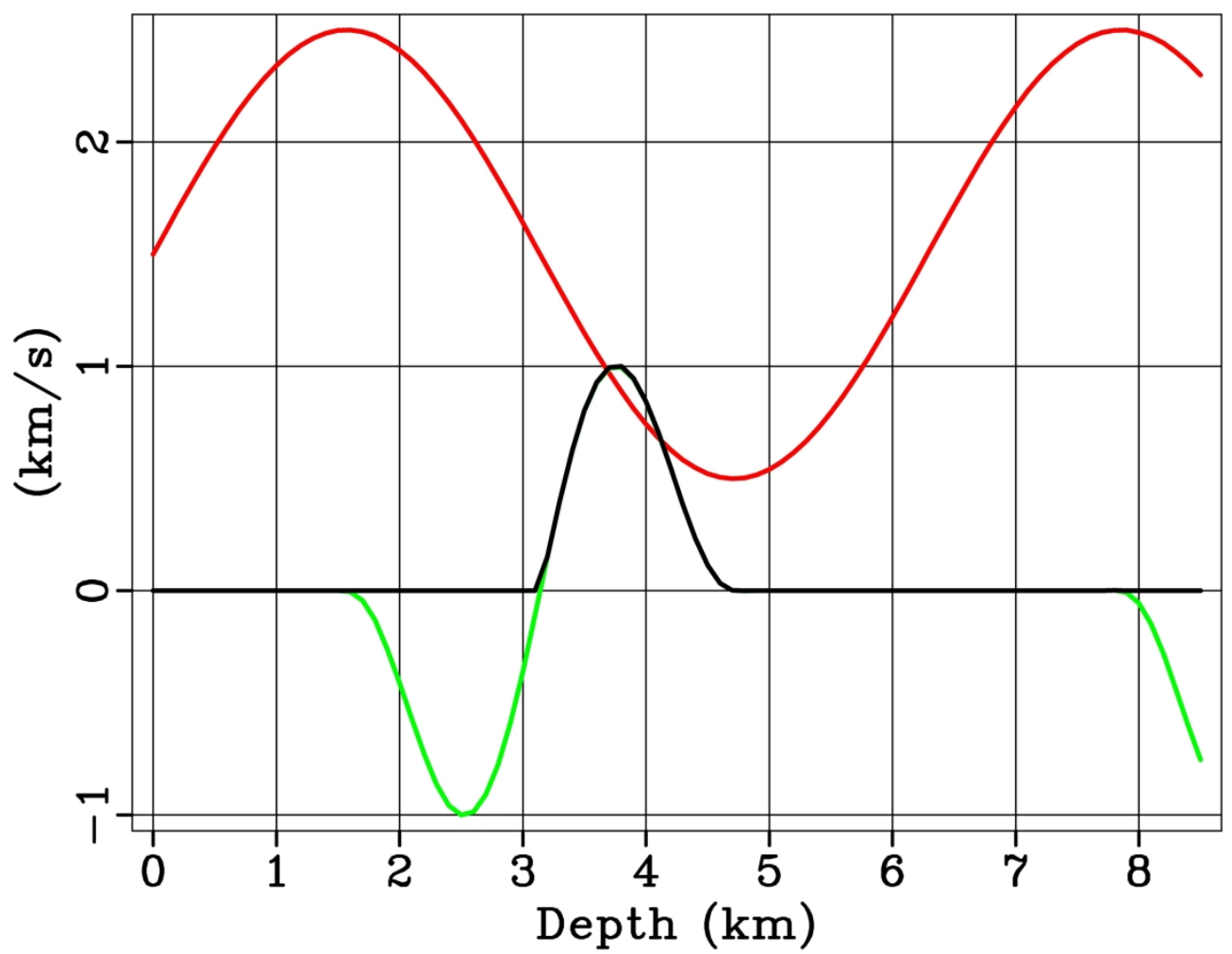

Figure 1: A dummy velocity profile (red); its update direction (green) is not free from undesired directions; the black line shows the controlled update.

$293 \times 228 \mathrm{~mm}(300 \times 300 \mathrm{DPI})$

This paper presented here as accepted for publication in Geophysics prior to copyediting and composition.

(C) 2019 Society of Exploration Geophysicists. 


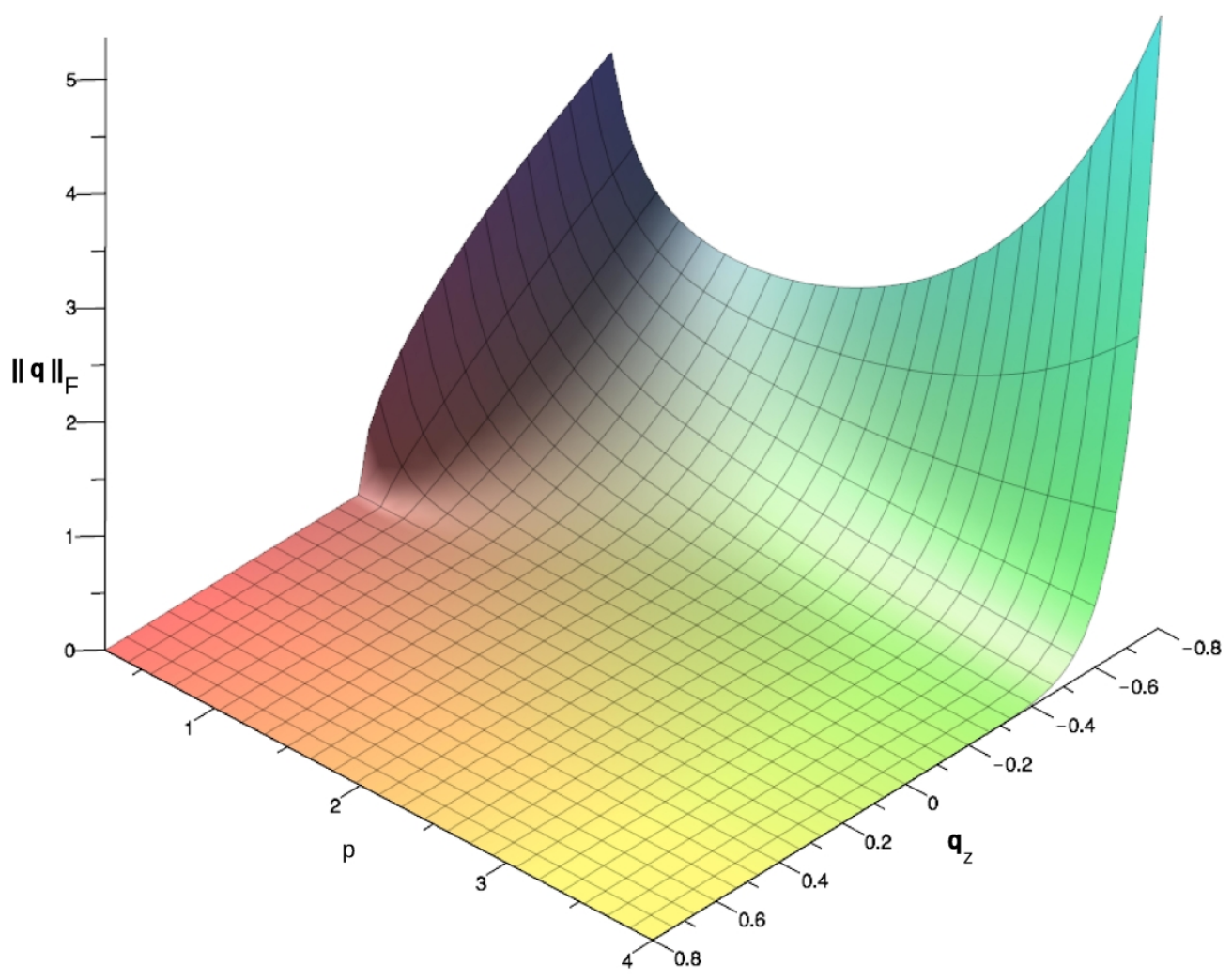

Figure 2: Behavior of the functional || q || $\mathrm{F}$ with $\mathrm{p}$. At large $\mathrm{p}$, the functional is biased towards steeper velocity drops compared to smaller ones.

$321 \times 259 \mathrm{~mm}(300 \times 300$ DPI $)$

This paper presented here as accepted for publication in Geophysics prior to copyediting and composition. (C) 2019 Society of Exploration Geophysicists. 


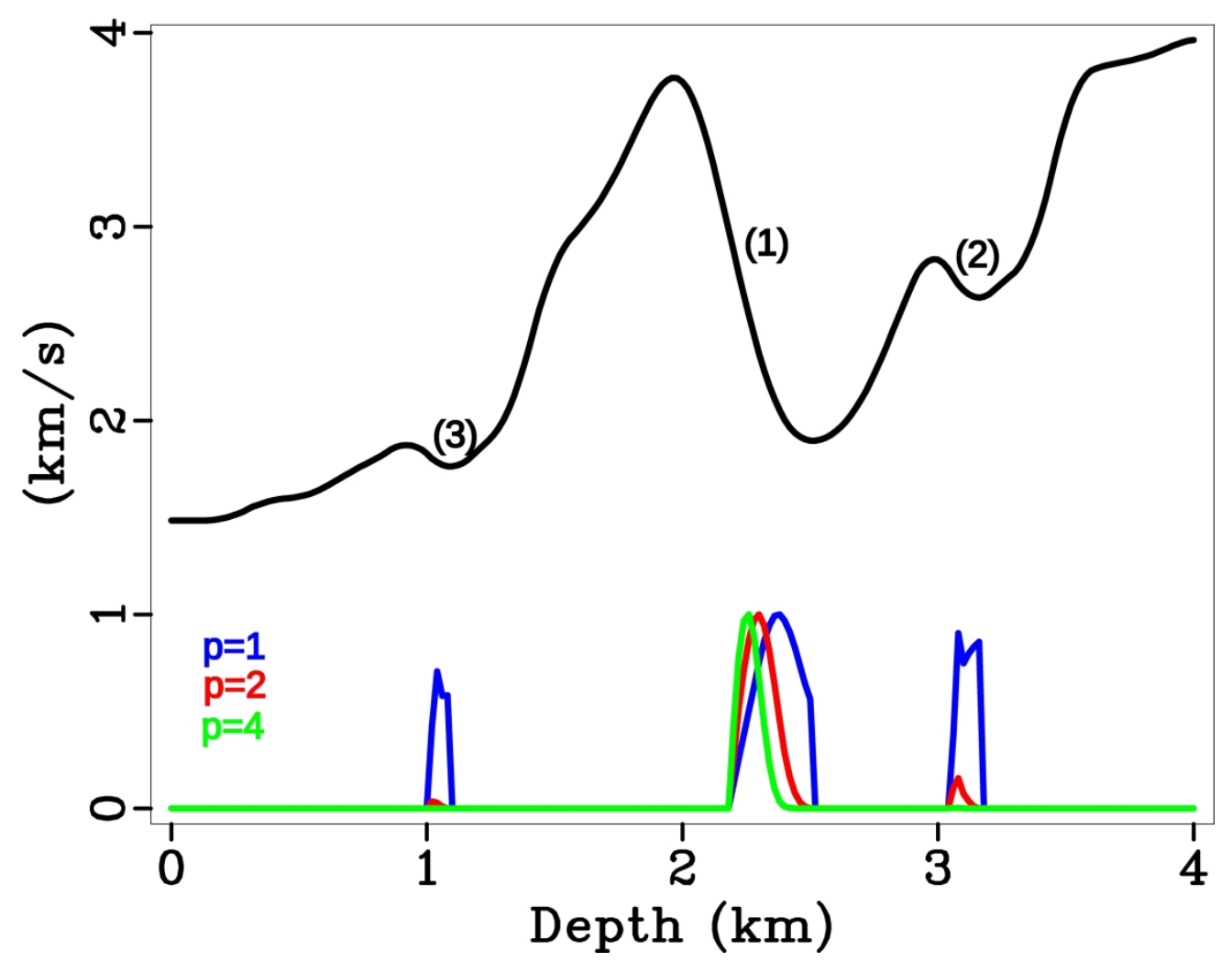

Figure 3: A dummy velocity profile (black) and its update directions with $p=\{1,2,4\}$. The large $\mathrm{p}$ makes the update direction immune to small-scale changes in velocity.

$291 \times 228 \mathrm{~mm}(300 \times 300$ DPI) 


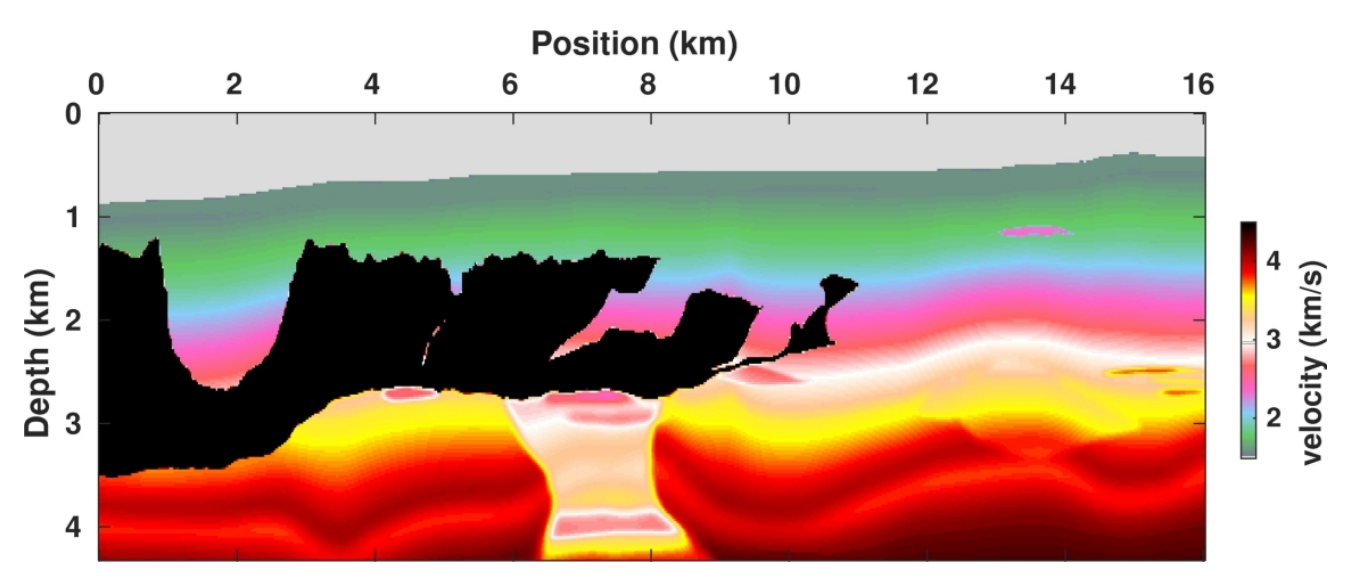

Figure 4: The west part of the BP 2004 velocity model.

$185 \times 75 \mathrm{~mm}(300 \times 300 \mathrm{DPI})$ 


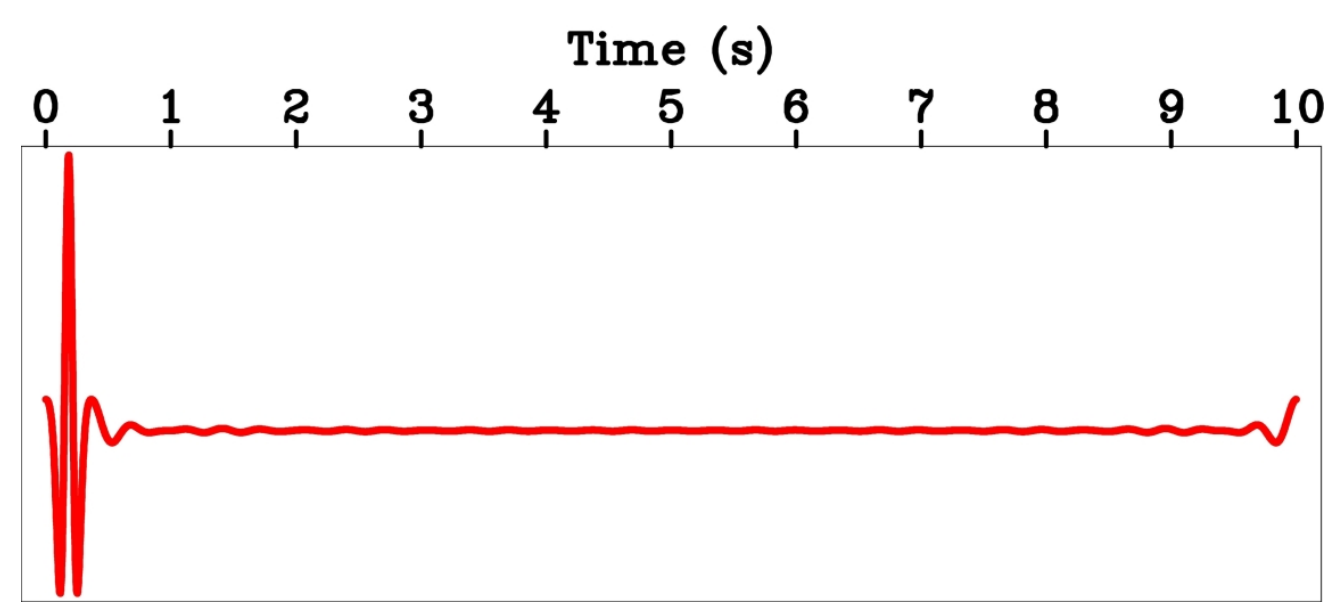

Figure 5: (a) The Ricker wavelet and

$272 \times 120 \mathrm{~mm}(300 \times 300$ DPI $)$

This paper presented here as accepted for publication in Geophysics prior to copyediting and composition.

(C) 2019 Society of Exploration Geophysicists. 
Frequency (Hz)

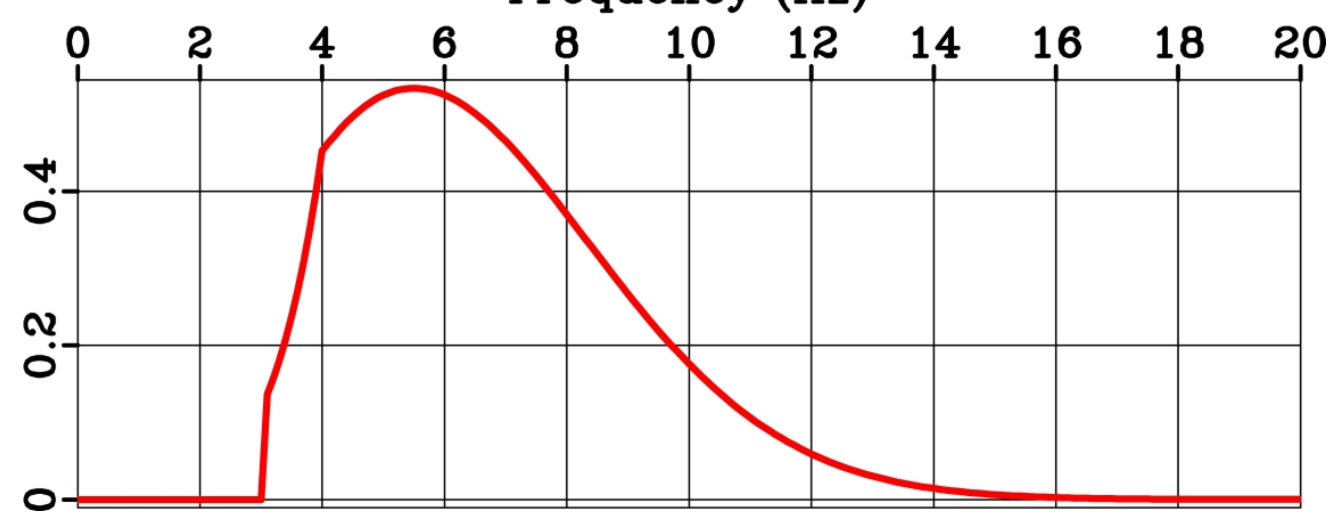

5(b) its frequency content in order to simulate the observed dataset. The lowest available frequency in the wavelet is $3.0 \mathrm{~Hz}$.

$289 \times 121 \mathrm{~mm}(300 \times 300 \mathrm{DPI})$ 


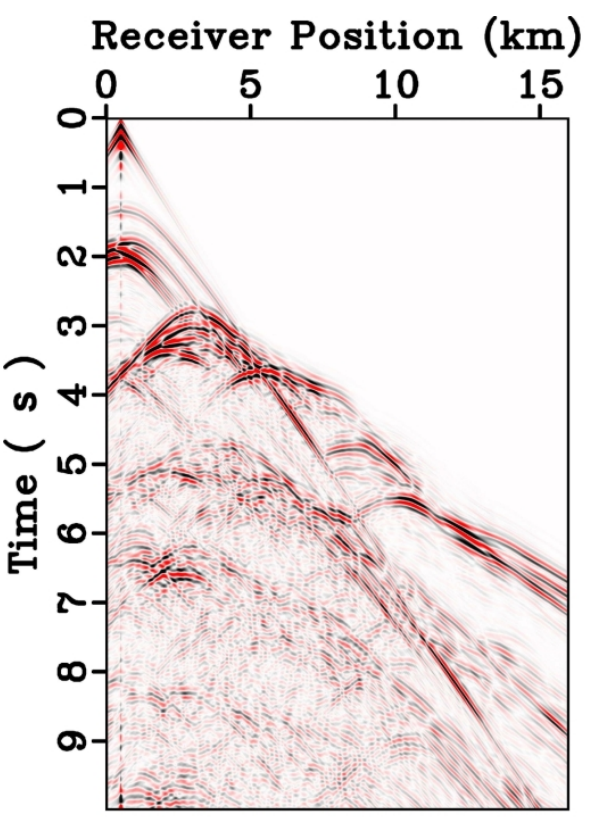

(a)

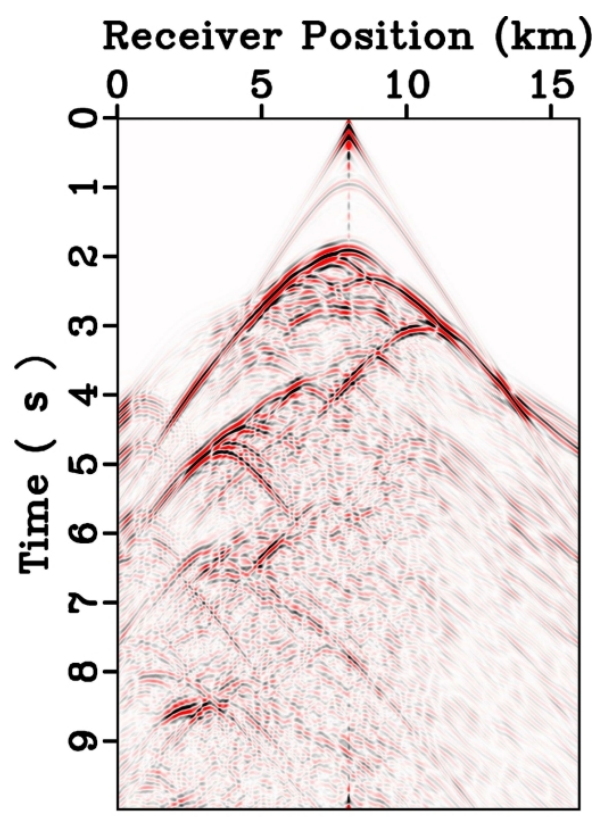

(b)

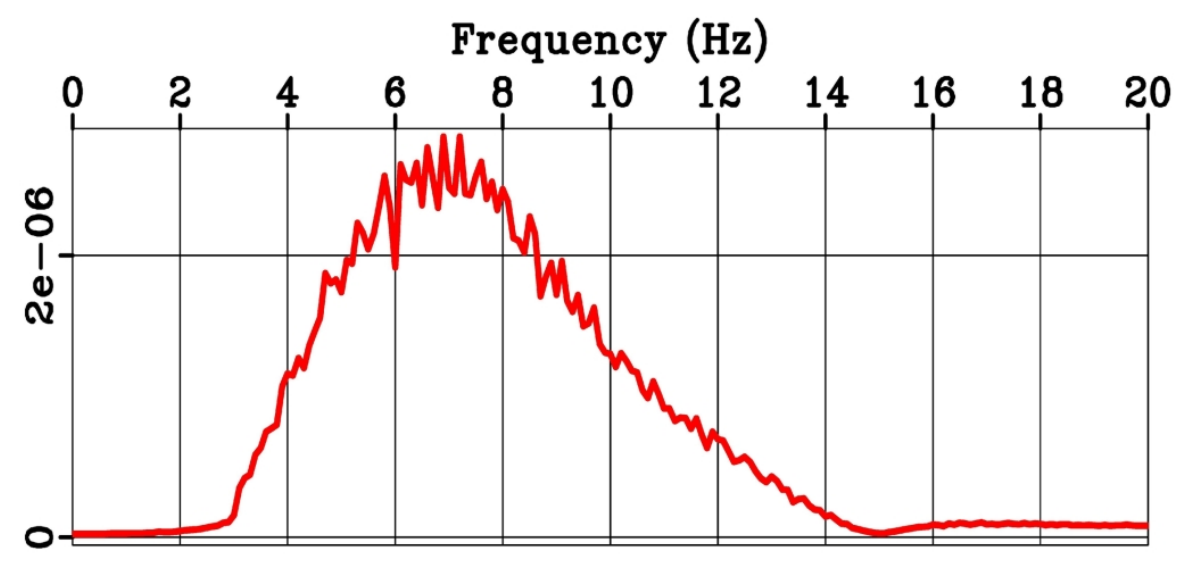

(c)

Figure 6: Shot records from the sources placed at (a) $x=0.5 \mathrm{~km}$ and (b) $x=8.0 \mathrm{~km}$, respectively; (c) average spectrum of (b).

$195 \times 259 \mathrm{~mm}(300 \times 300$ DPI $)$ 


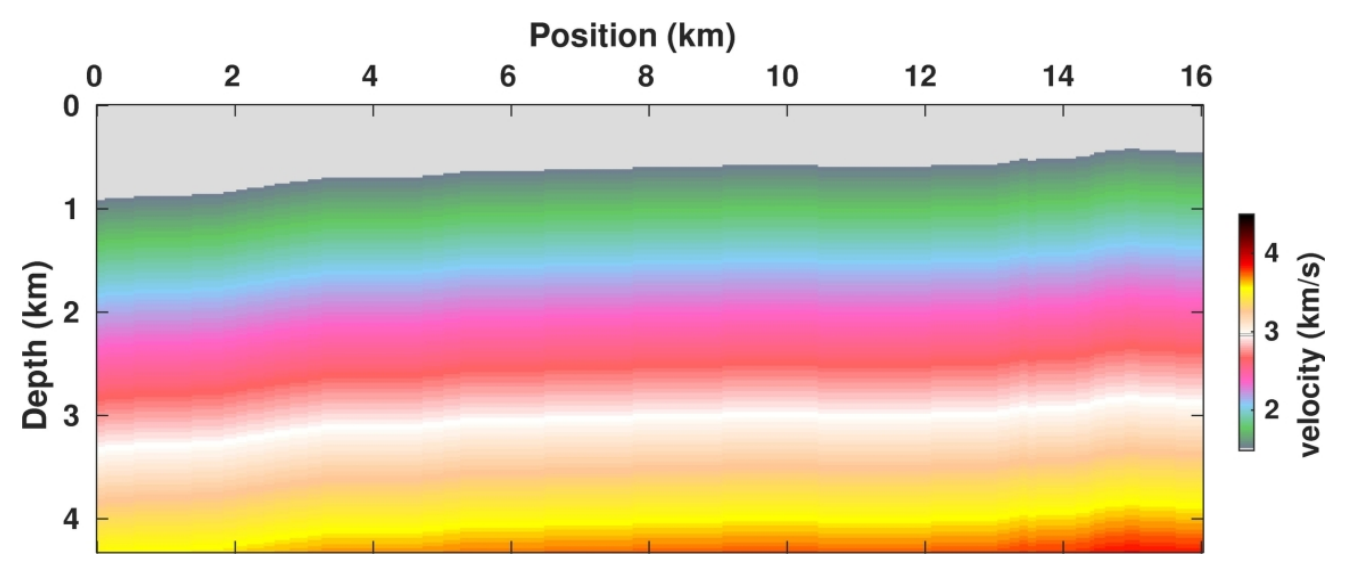

Figure 7: Initial model to invert for the BP 2004 velocity.

$185 \times 75 \mathrm{~mm}(300 \times 300 \mathrm{DPI})$ 


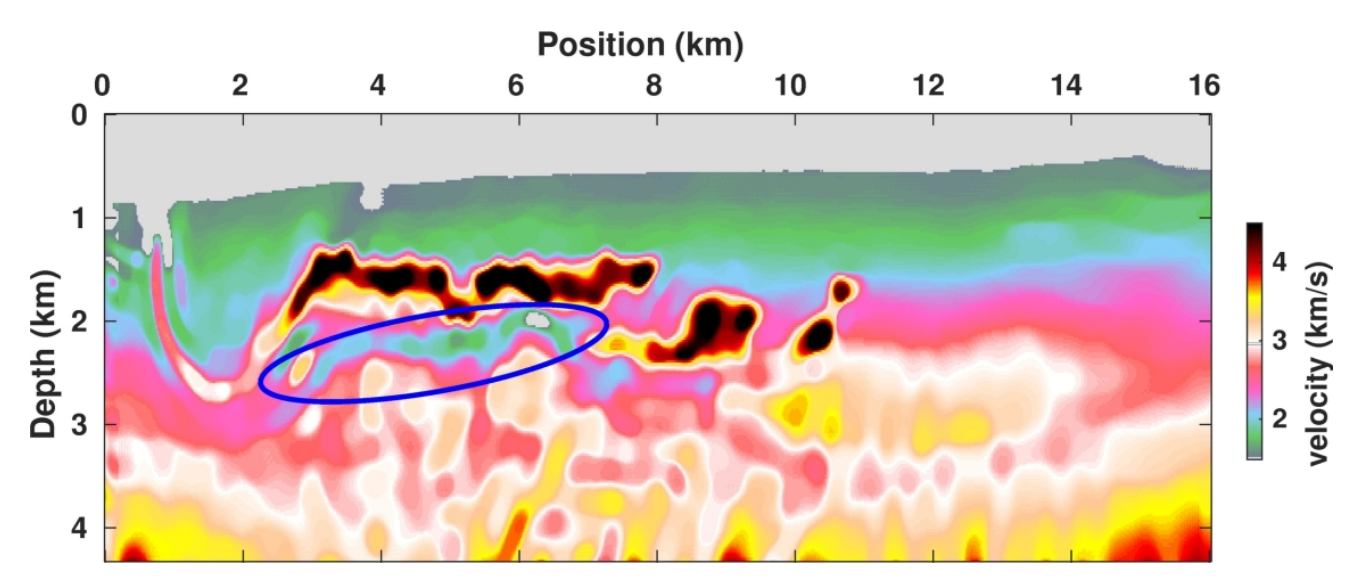

Figure 8: Results of the first optimization problem ('FWI + TV'). The blue circle indicates the region where FWI starts failing to update the velocity in the right direction.

\section{$185 \times 75 \mathrm{~mm}(300 \times 300 \mathrm{DPI})$}



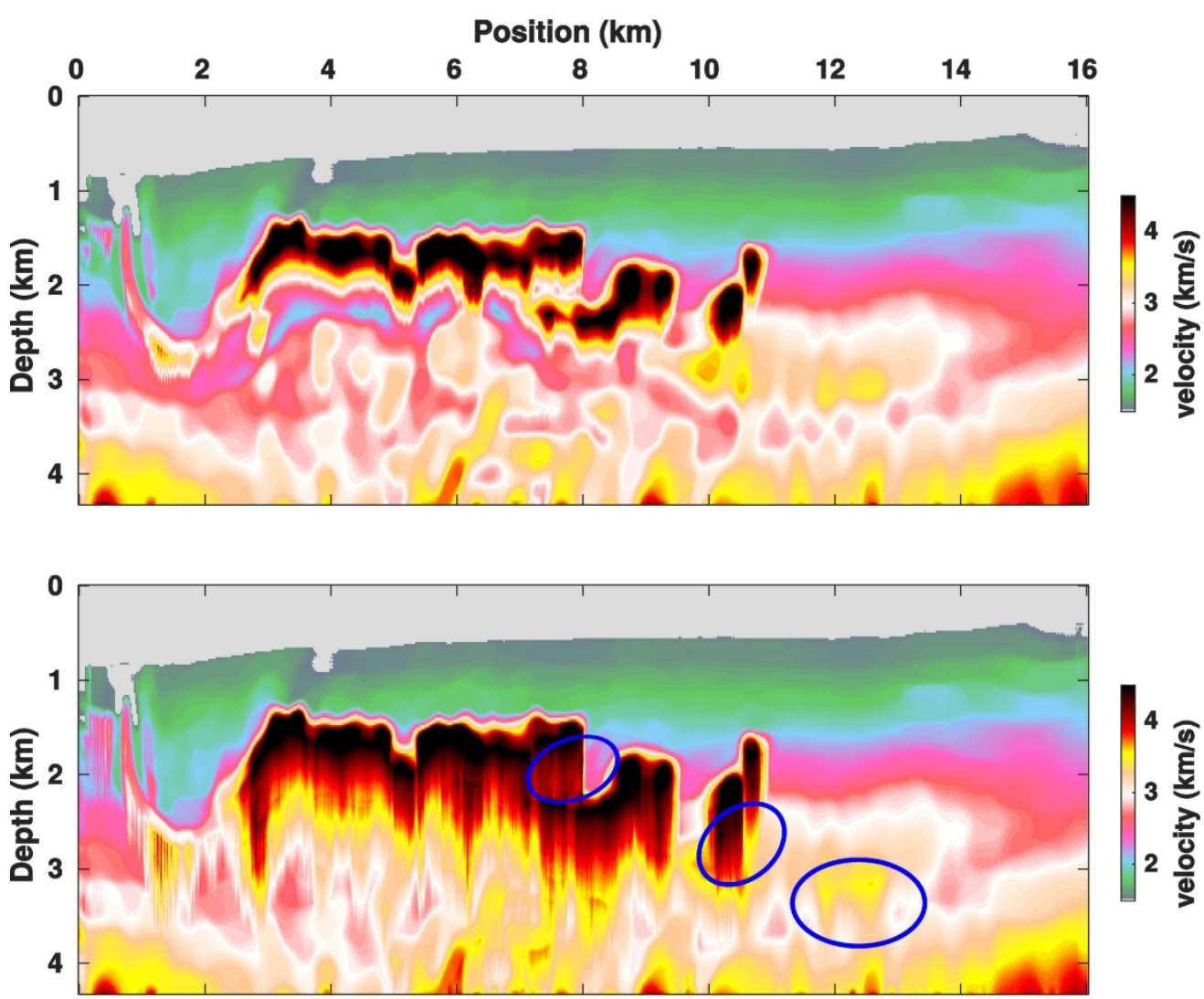

Figure 9: Snapshots from the second optimization problem ('flooding'). The flooding procedure succeeded in wiping out the wrong velocity of the reconstructed model of 'FWI+TV' (Figure 8). However, we observe undue flooding in some regions, which is indicated by the ellipses in the bottom panel.

$185 \times 152 \mathrm{~mm}(300 \times 300 \mathrm{DPI})$ 


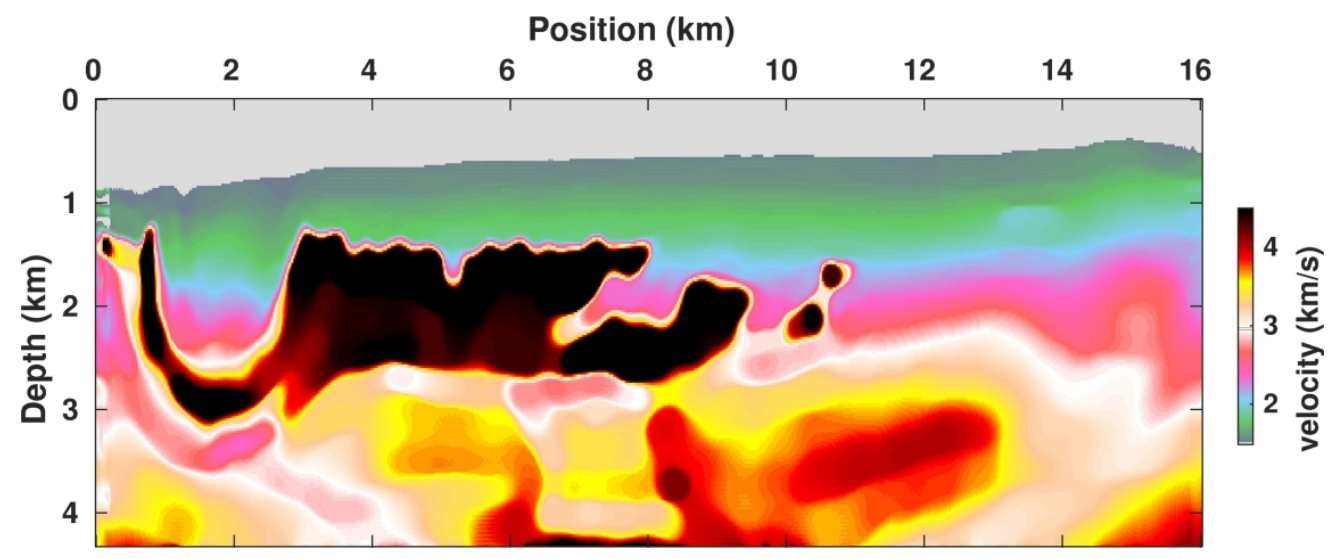

Figure 10: Output of the 'FWI+TV' scheme with the input velocity from the bottom panel of Figure 9. 'FWI+TV' removed the residuals of undue flooding, and recovered most, including the subsalt, regions of the model.

$$
185 \times 75 \mathrm{~mm}(300 \times 300 \mathrm{DPI})
$$




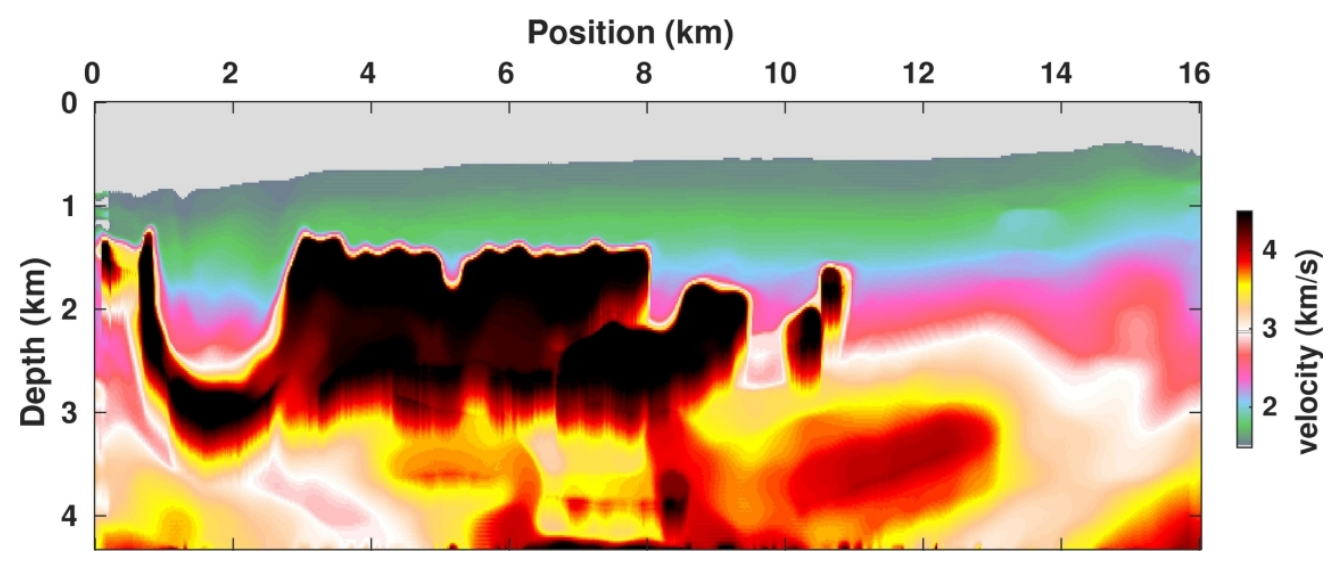

Figure 11: Output of the 'FWI+TV' scheme with the input velocity from the bottom panel of Figure 9. 'FWI+TV' removed the residuals of undue flooding, and recovered most, including the subsalt, regions of the model.

$$
185 \times 75 \mathrm{~mm}(300 \times 300 \mathrm{DPI})
$$



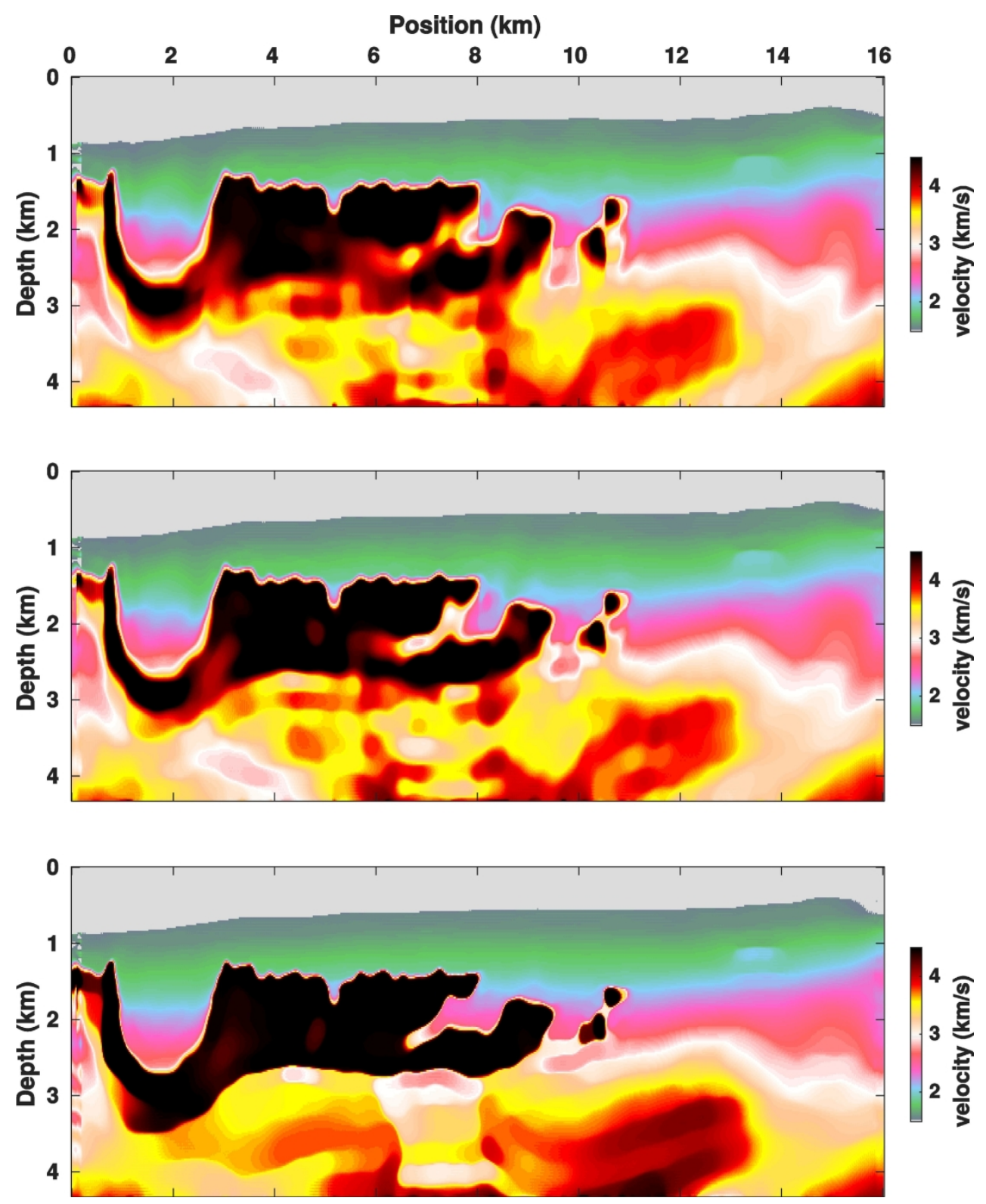

Figure 12: (From top to bottom) Evolution of the velocity model using 'FWI+TV'. We decrease the regularization coefficient of TV with higher frequency bandwidths.

$185 \times 228 \mathrm{~mm}(300 \times 300$ DPI $)$ 


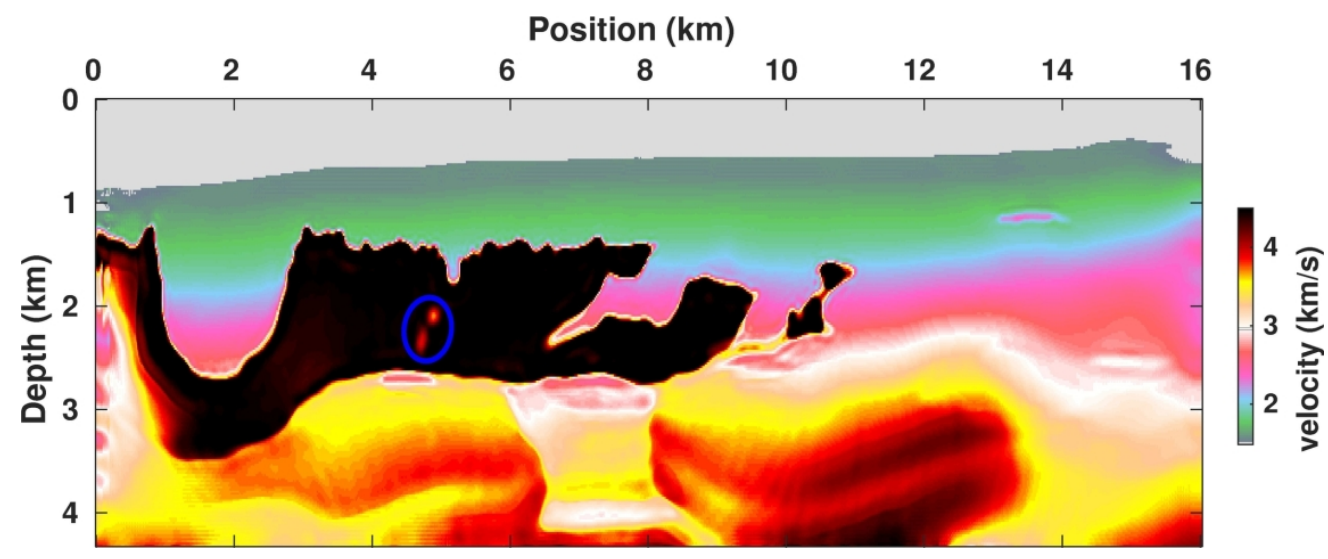

Figure 13: Final reconstructed model of 'FWI+TV' with $\lambda T V=0$ for the full bandwidth of the dataset. We retrieved not only the salt and subsalt regions, but also the dirty salt as indicated by the blue circle.

$$
185 \times 75 \mathrm{~mm}(300 \times 300 \mathrm{DPI})
$$




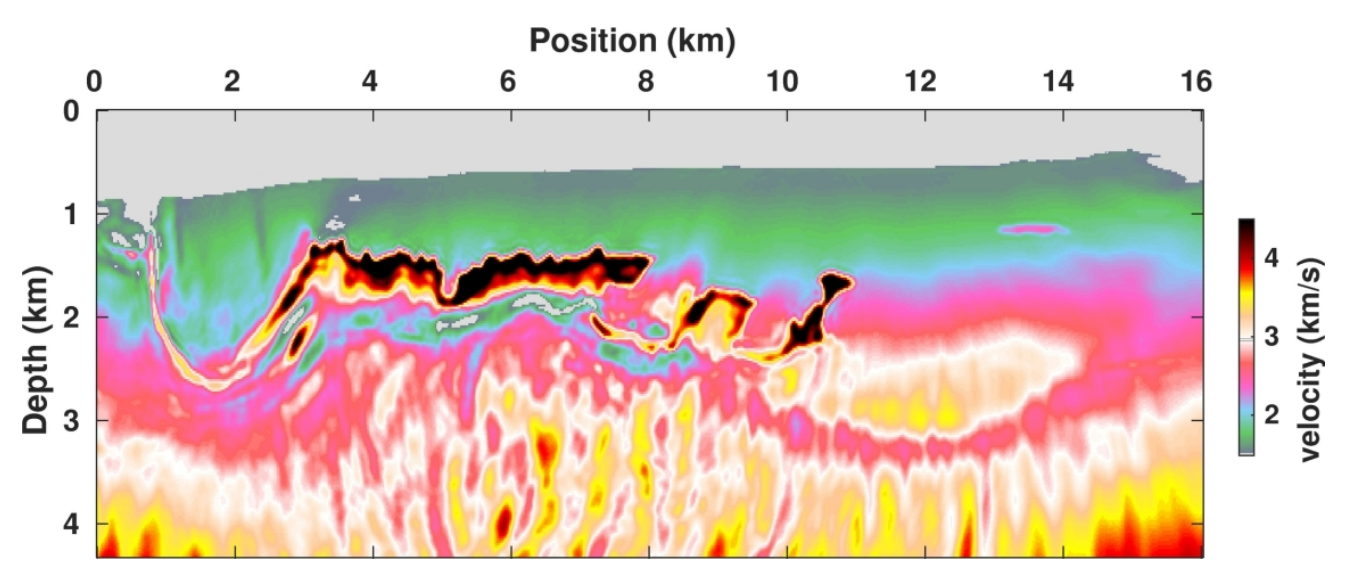

Figure 14: Reconstructed model with no regularization, i.e., conventional FWI with multi-scale approach (Bunks et al., 1995). Compare with Figure 13.

$185 \times 75 \mathrm{~mm}(300 \times 300 \mathrm{DPI})$ 


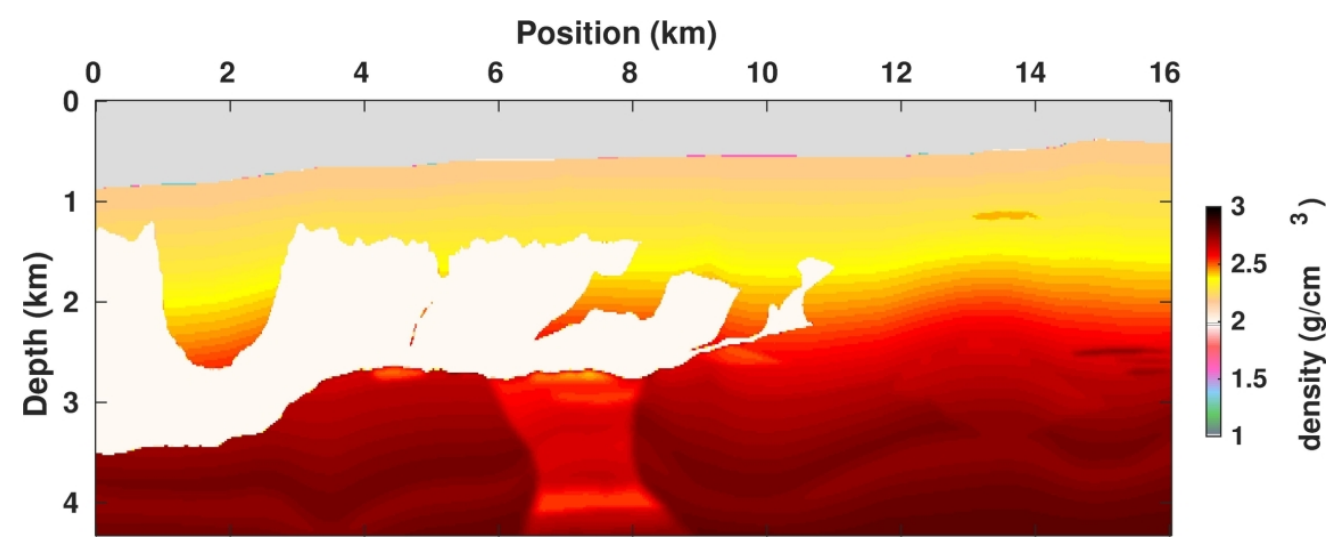

Figure 15: Variable density of the BP 2004 model. The density of the salt body is lower than that of the sedimentary neighborhood.

$190 \times 75 \mathrm{~mm}(300 \times 300$ DPI $)$ 


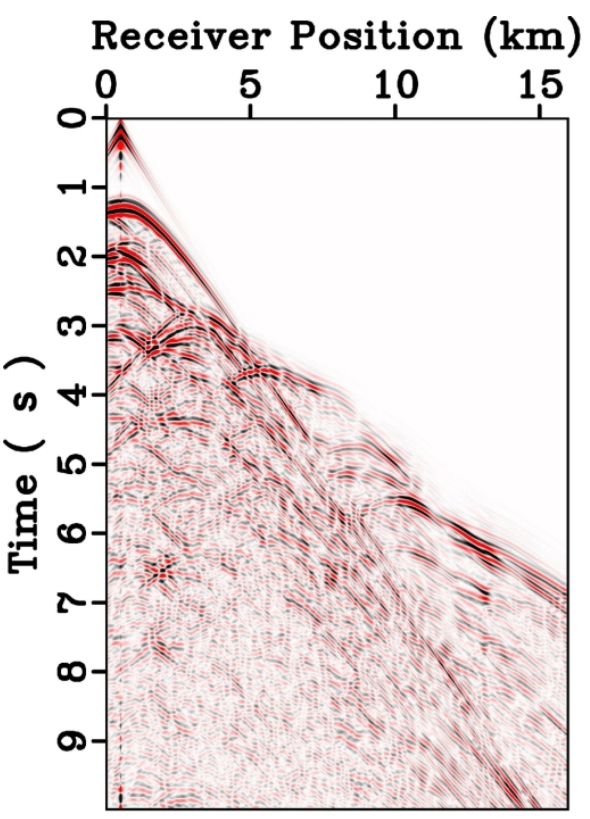

(a)

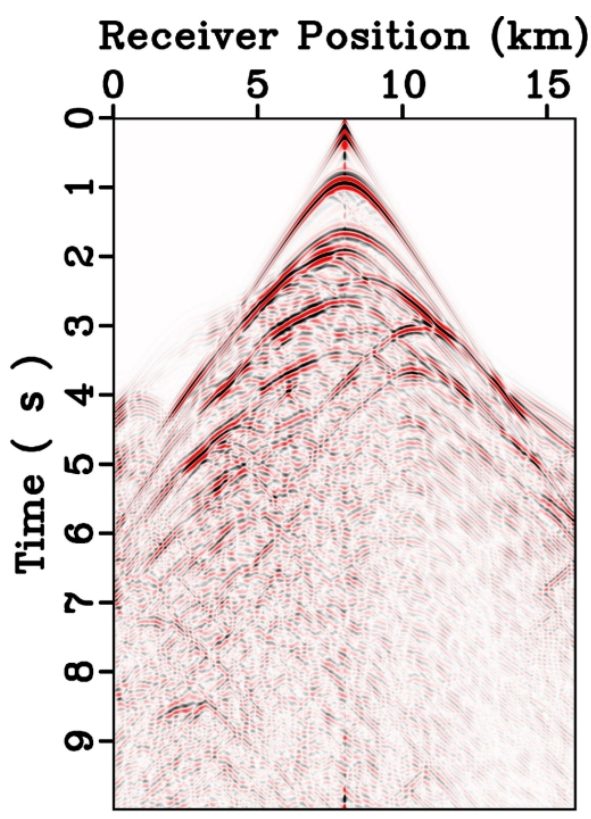

(b)

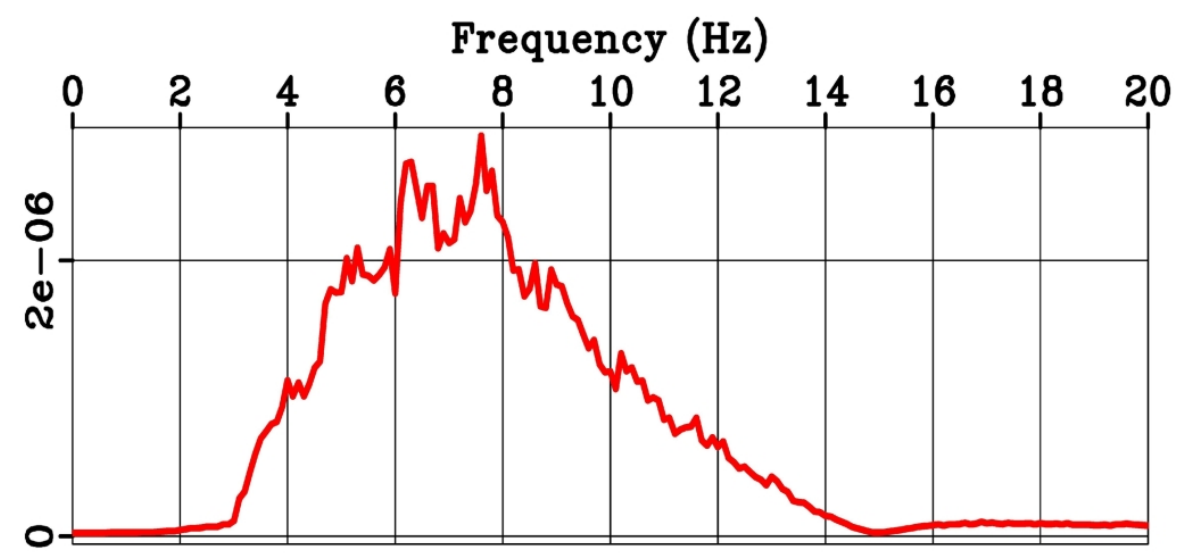

(c)

Figure 16: Simulated observed data using acoustic wave equation with variable density. The shot gathers for the sources placed at (a) $x=0.5 \mathrm{~km}$ and (b) $x=8.0 \mathrm{~km}$, respectively; (c) average frequency spectrum of data shown in (b). Compare with Figure 6.

$195 \times 259 \mathrm{~mm}(300 \times 300$ DPI) 


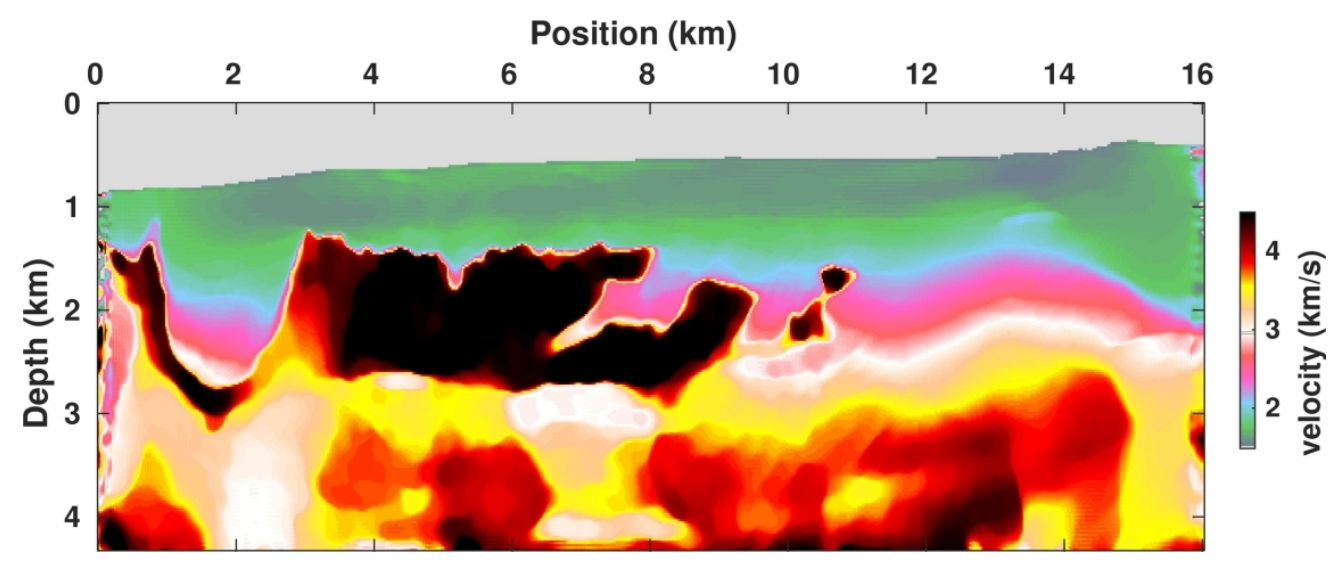

Figure 17: Final velocity model reconstructed from the observed dataset, displayed in Figure 16.

$185 \times 75 \mathrm{~mm}(300 \times 300 \mathrm{DPI})$ 


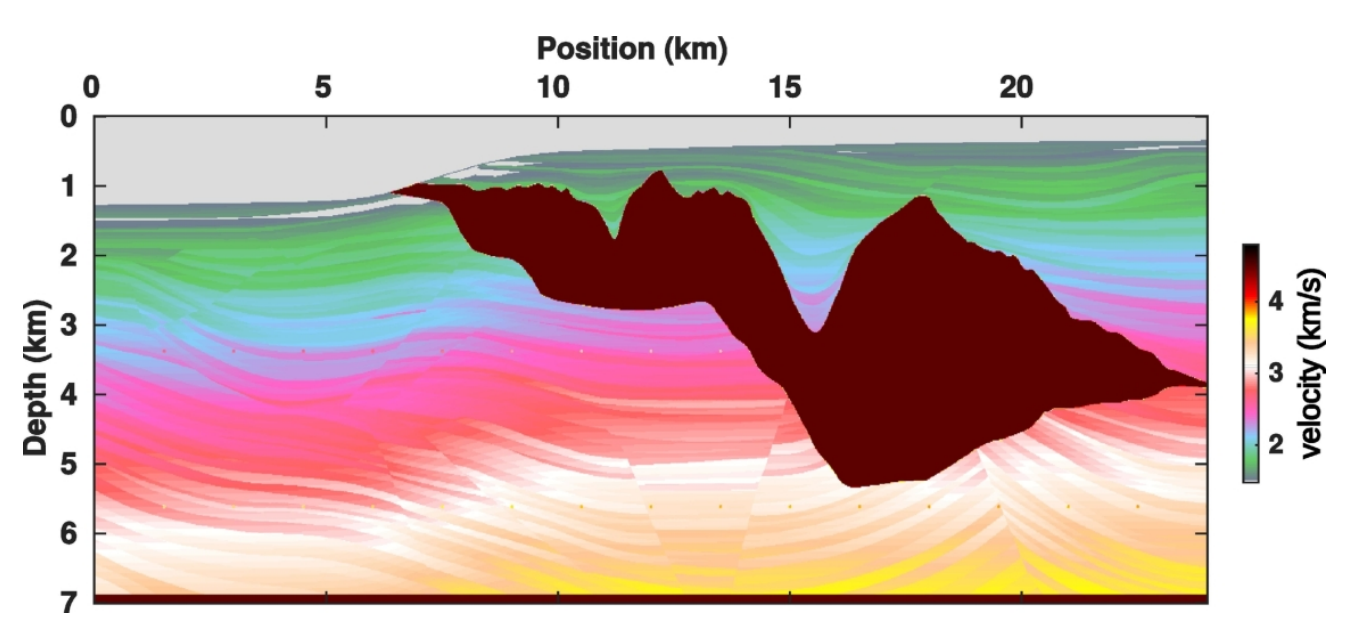

Figure 18: Sigsbee2A velocity model (Paffenholz et al., 2002).

$184 \times 81 \mathrm{~mm}(300 \times 300 \mathrm{DPI})$ 


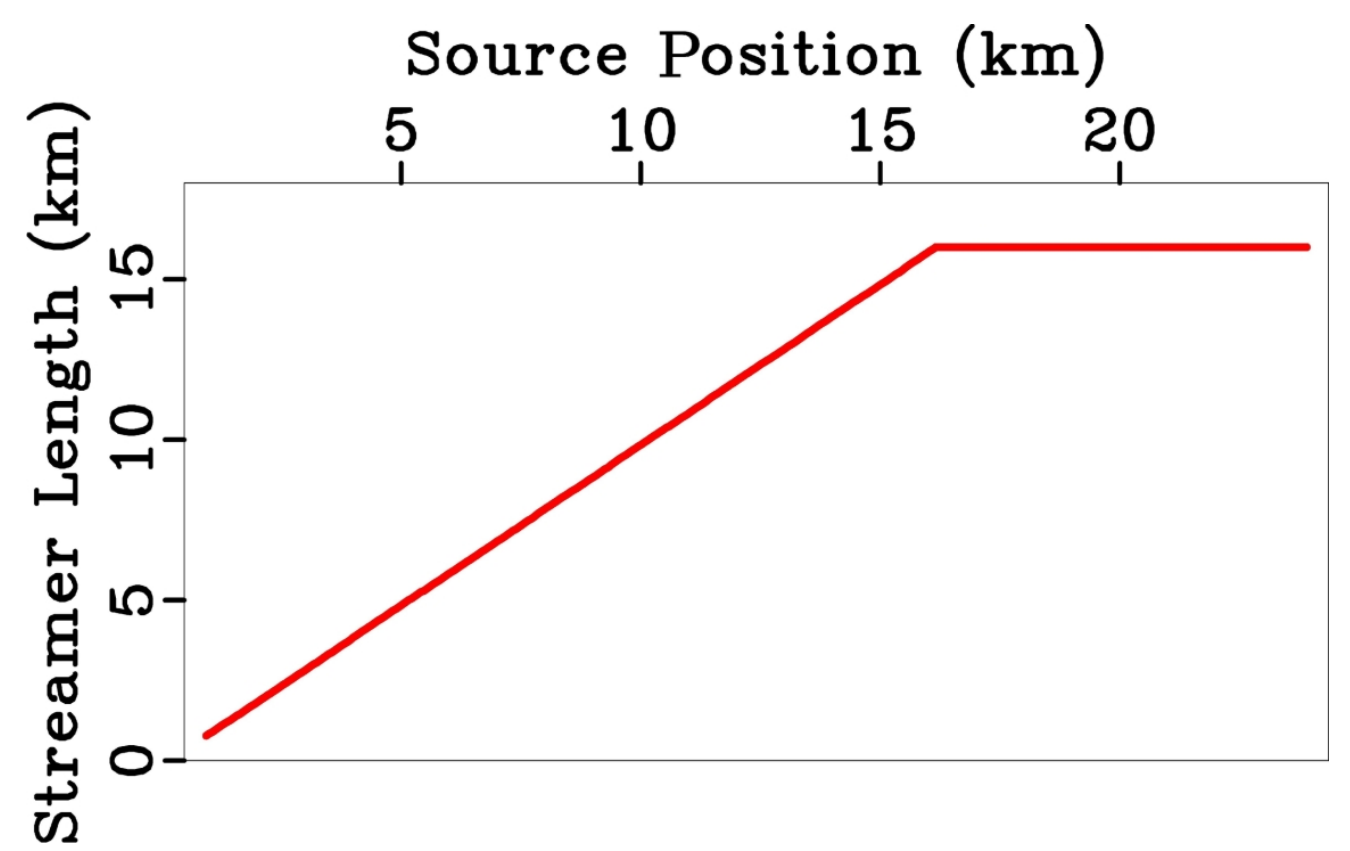

Figure 19: Available streamer length for each shot (maximum $=16.0 \mathrm{~km}$, minimum $=0.8 \mathrm{~km}$ ). The acquisition coverage towards the left part of the model is very poor.

$203 \times 128 \mathrm{~mm}(300 \times 300 \mathrm{DPI})$ 


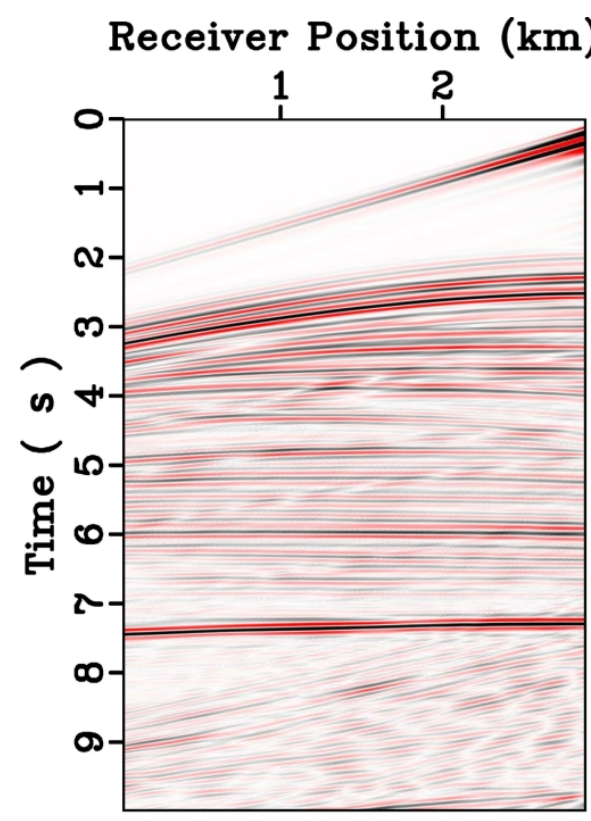

(a)

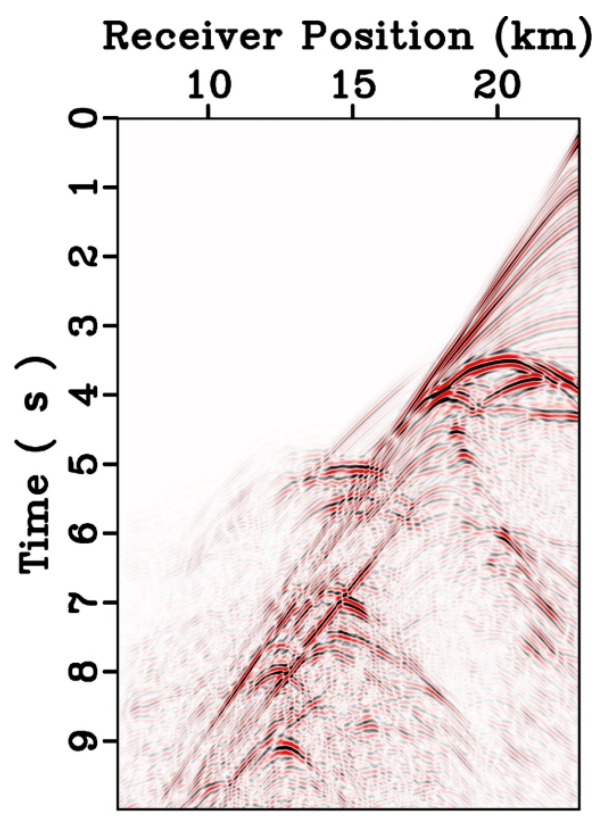

(b)

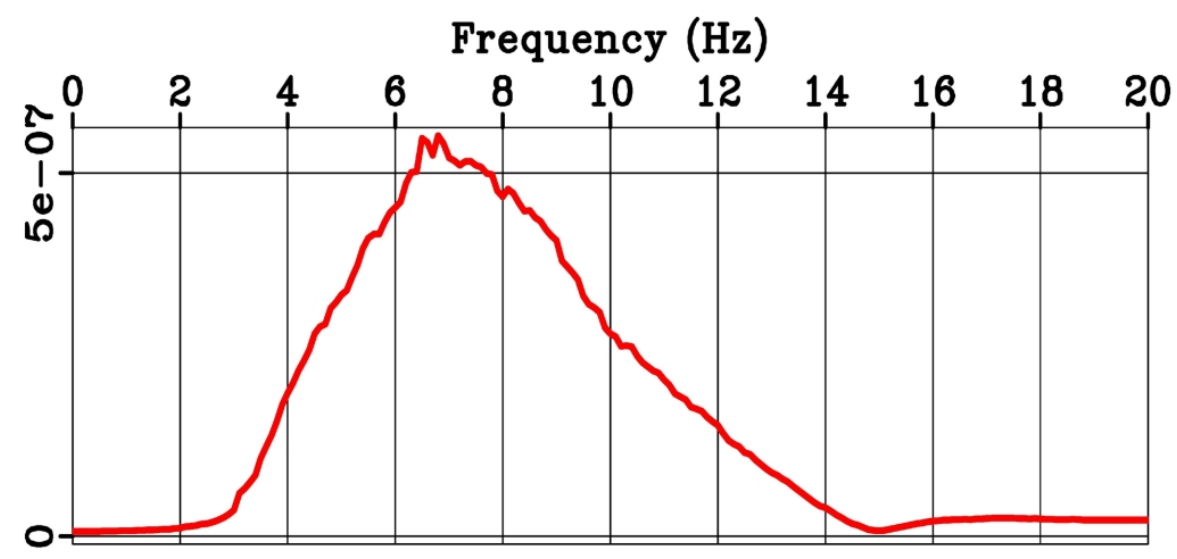

(c)

Figure 20: Shot gathers from the sources placed at (a) $x=2.9 \mathrm{~km}$ and (b) $x=23.0 \mathrm{~km}$; (c) average frequency spectrum of the data shown in (b).

$$
195 \times 259 \mathrm{~mm}(300 \times 300 \mathrm{DPI})
$$



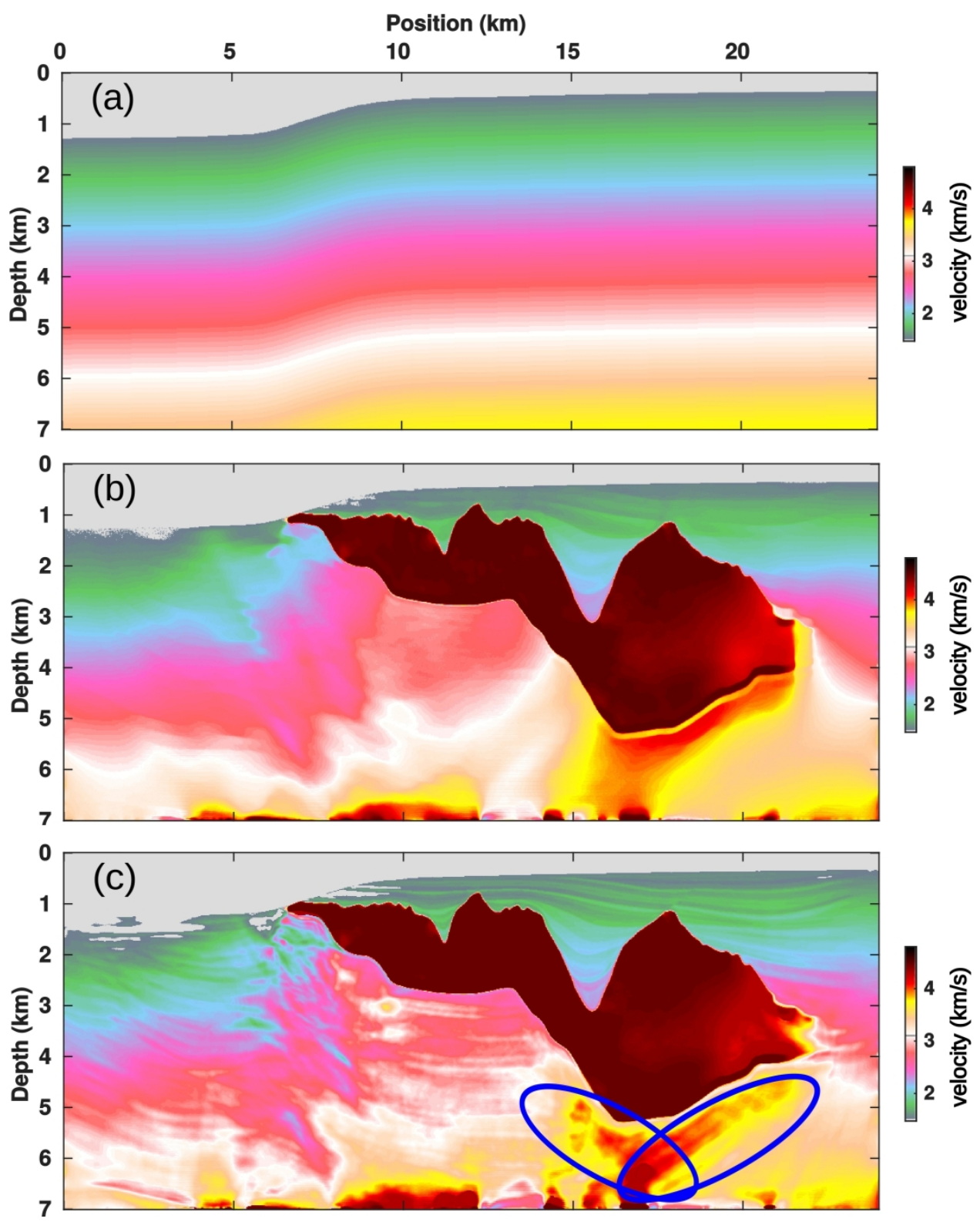

Figure 21: (a) Initial model, (b) inverted model, and (c) final inverted model using Figure 21(b) as the initial model, with the gradient preconditioned to zero whenever the velocity is larger than 4.2 $\mathrm{km} / \mathrm{s}$. The inversion process could not remove the unwanted over-flooding from the subsalt region as indicated by the ellipses.

$185 \times 232 \mathrm{~mm}(300 \times 300 \mathrm{DPI})$ 

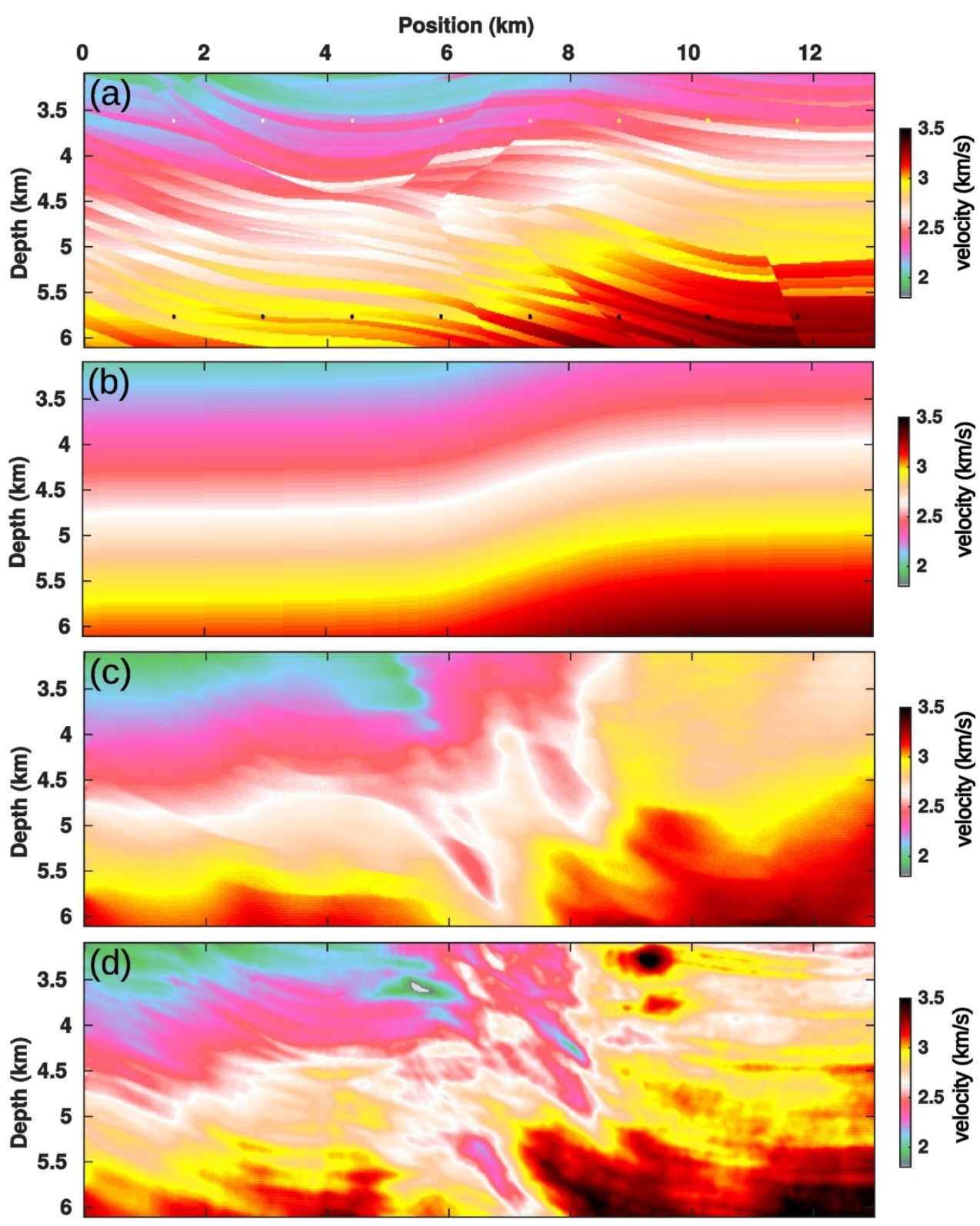

Figure 22: Enlarged sections of the (a) true, (b) initial, (c) intermediate (Figure 21(b)), and (d) final inverted (Figure 21(c)) models.

$192 \times 239 \mathrm{~mm}(300 \times 300 \mathrm{DPI})$ 


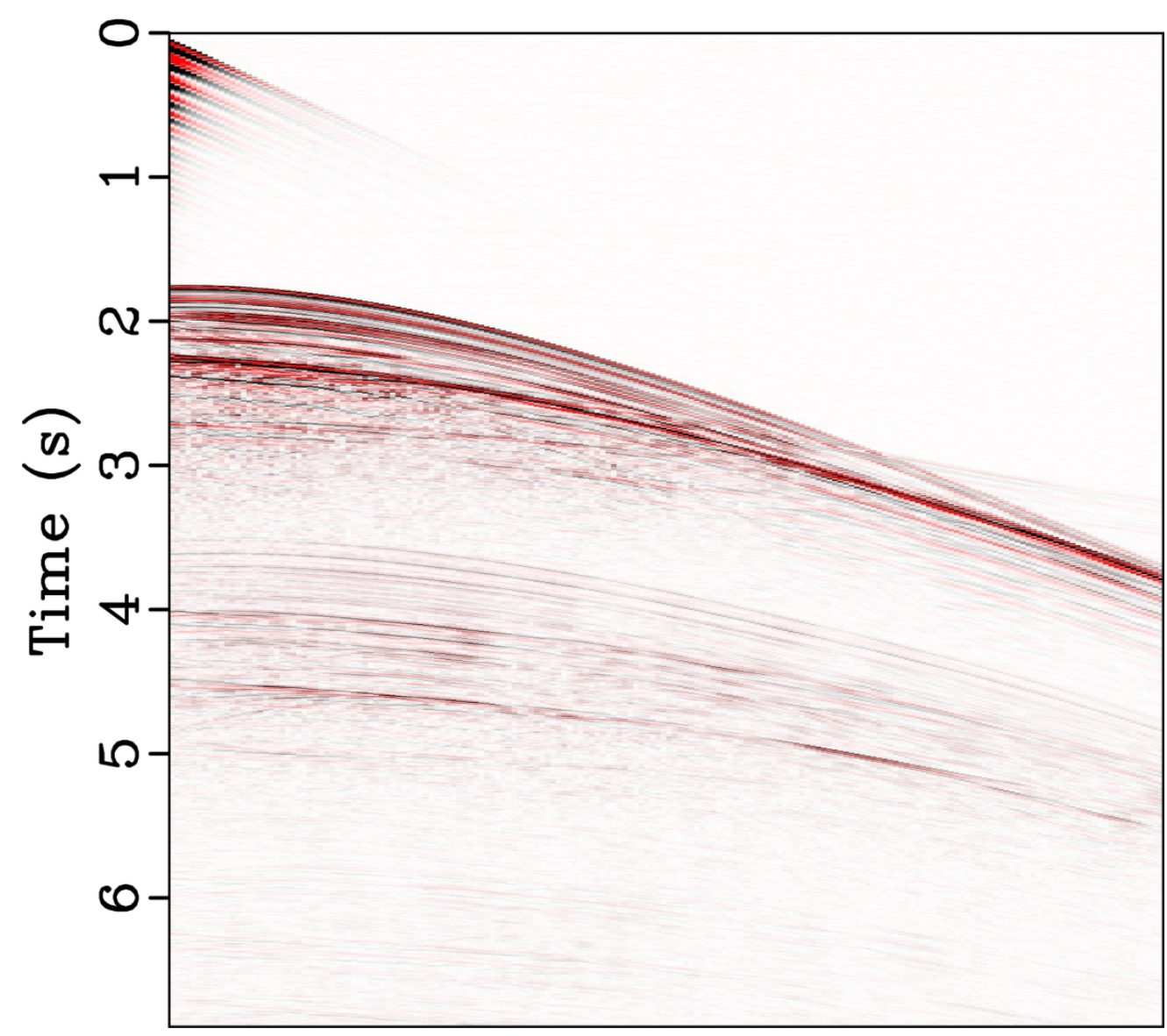

Figure 23a: (a) A shot gather from the source placed at $x=11.0 \mathrm{~km}$ and (b) its frequency content. There is no useful signal below $7 \mathrm{~Hz}$.

$244 \times 216 \mathrm{~mm}(300 \times 300 \mathrm{DPI})$

This paper presented here as accepted for publication in Geophysics prior to copyediting and composition. (C) 2019 Society of Exploration Geophysicists. 


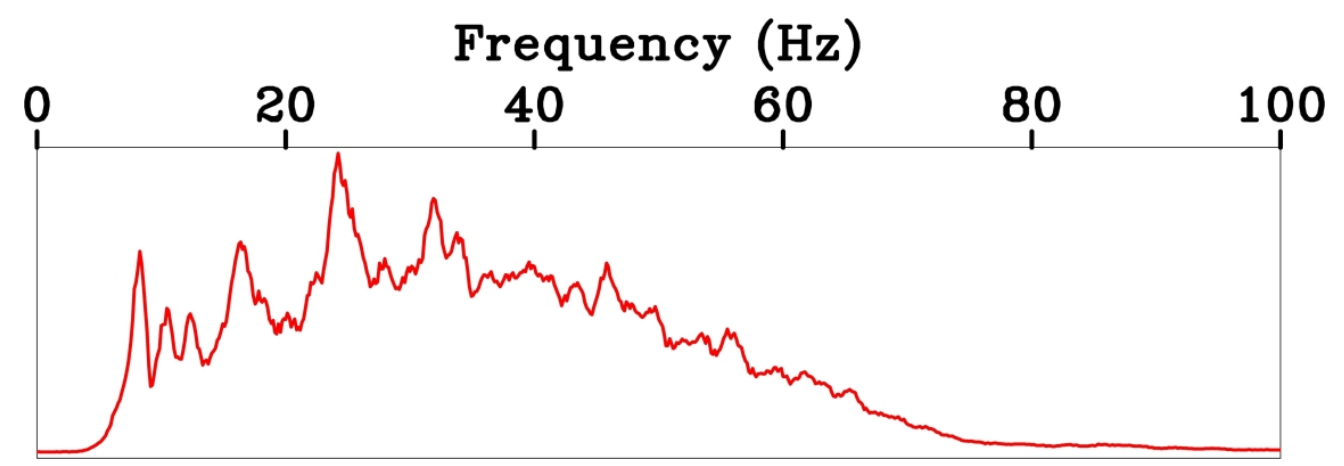

Figure 23: (a) A shot gather from the source placed at $x=11.0 \mathrm{~km}$ and (b) its frequency content. There is no useful signal below $7 \mathrm{~Hz}$.

$318 \times 108 \mathrm{~mm}(300 \times 300 \mathrm{DPI})$ 


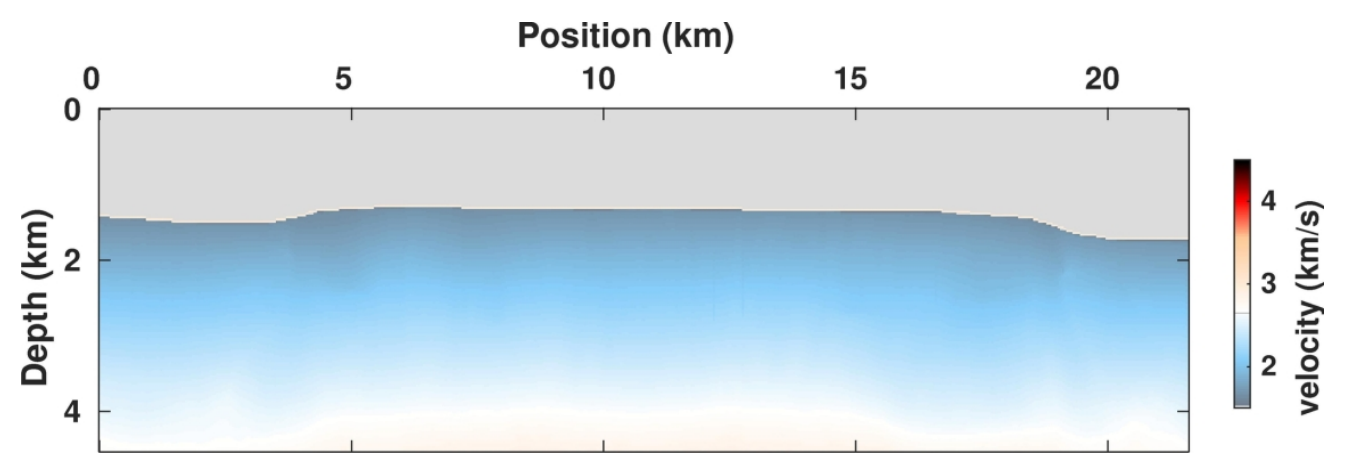

Figure 24: Initial velocity model to invert available GOM dataset.

$176 \times 58 \mathrm{~mm}(300 \times 300 \mathrm{DPI})$

This paper presented here as accepted for publication in Geophysics prior to copyediting and composition. (C) 2019 Society of Exploration Geophysicists. 


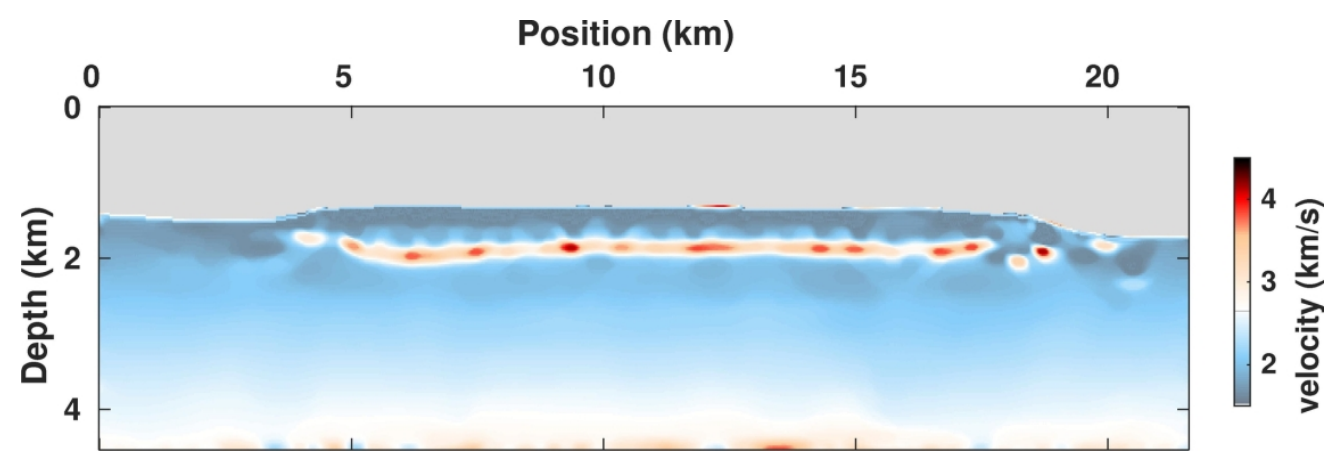

Figure 25P1: Evolution of the velocity model through our proposed method: the left panel shows the output of 'FWI+TV' scheme, which are subjected to 'flooding' procedure to obtain the right panel. For 'flooding', we assign $\mathrm{p}=4$.

$176 \times 58 \mathrm{~mm}(300 \times 300 \mathrm{DPI})$ 


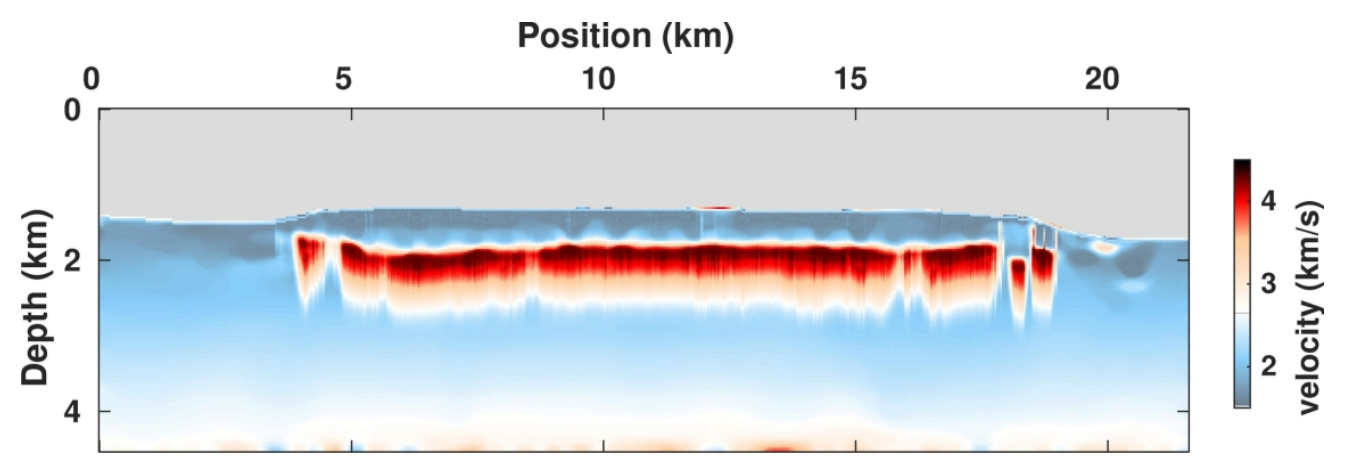

Figure 25P2: Evolution of the velocity model through our proposed method: the left panel shows the output of 'FWI+TV' scheme, which are subjected to 'flooding' procedure to obtain the right panel. For 'flooding', we assign $\mathrm{p}=4$.

$176 \times 58 \mathrm{~mm}(300 \times 300 \mathrm{DPI})$ 


\section{Position (km)}

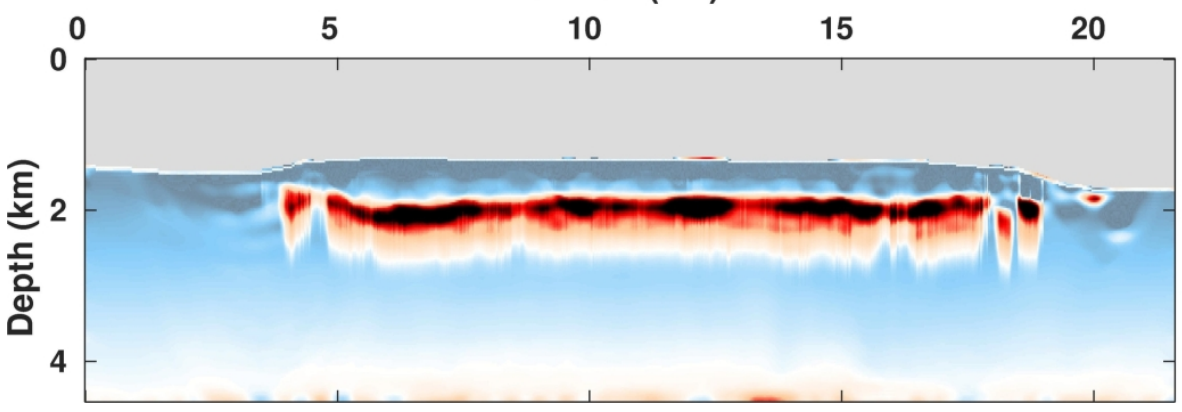

4

3 है

2

2 응

Figure 25P3: Evolution of the velocity model through our proposed method: the left panel shows the output of 'FWI+TV' scheme, which are subjected to 'flooding' procedure to obtain the right panel. For 'flooding', we assign $\mathrm{p}=4$.

$176 \times 58 \mathrm{~mm}(300 \times 300 \mathrm{DPI})$ 


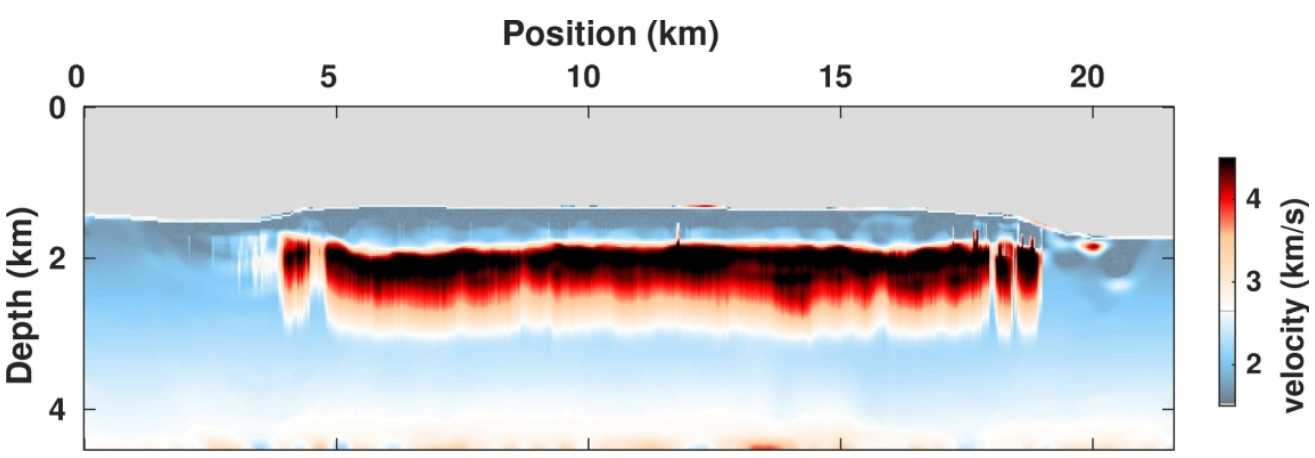

Figure 25P4: Evolution of the velocity model through our proposed method: the left panel shows the output of 'FWI+TV' scheme, which are subjected to 'flooding' procedure to obtain the right panel. For 'flooding', we assign $\mathrm{p}=4$.

$176 \times 58 \mathrm{~mm}(300 \times 300 \mathrm{DPI})$ 


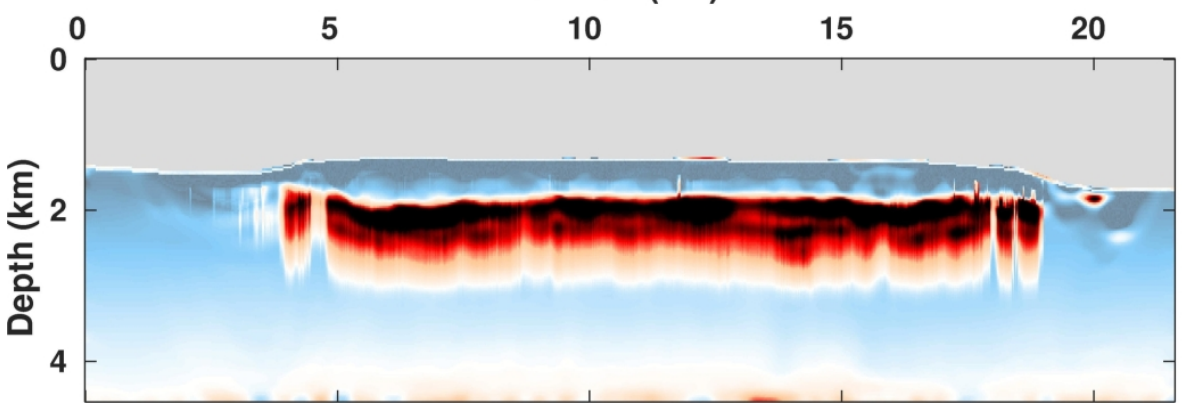

4

Figure 25P5: Evolution of the velocity model through our proposed method: the left panel shows the output of 'FWI+TV' scheme, which are subjected to 'flooding' procedure to obtain the right panel. For 'flooding', we assign $\mathrm{p}=4$.

$176 \times 58 \mathrm{~mm}(300 \times 300 \mathrm{DPI})$ 


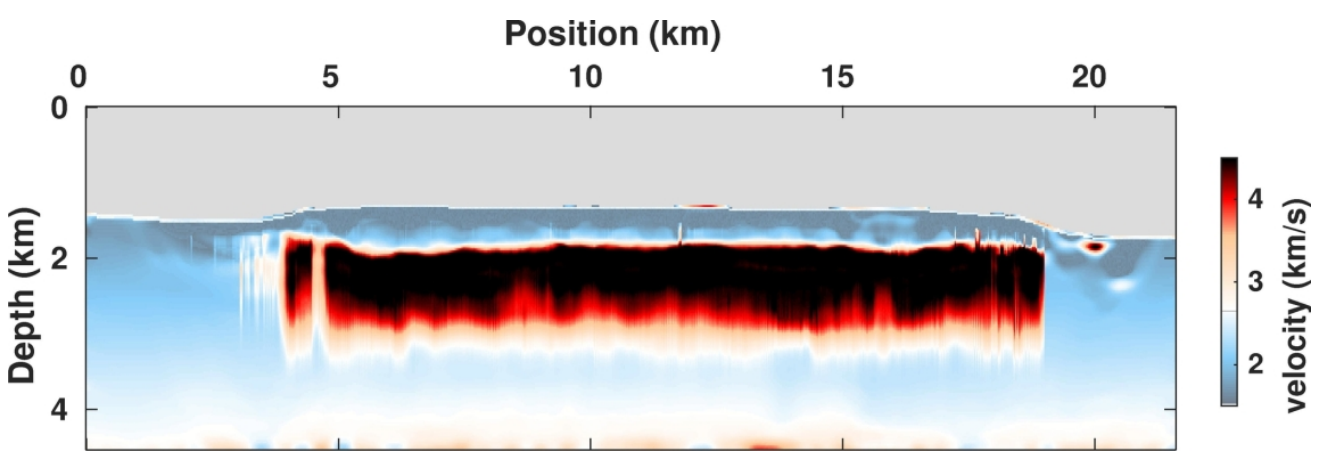

Figure 25P6: Evolution of the velocity model through our proposed method: the left panel shows the output of 'FWI+TV' scheme, which are subjected to 'flooding' procedure to obtain the right panel. For 'flooding', we assign $\mathrm{p}=4$.

$176 \times 58 \mathrm{~mm}(300 \times 300$ DPI) 


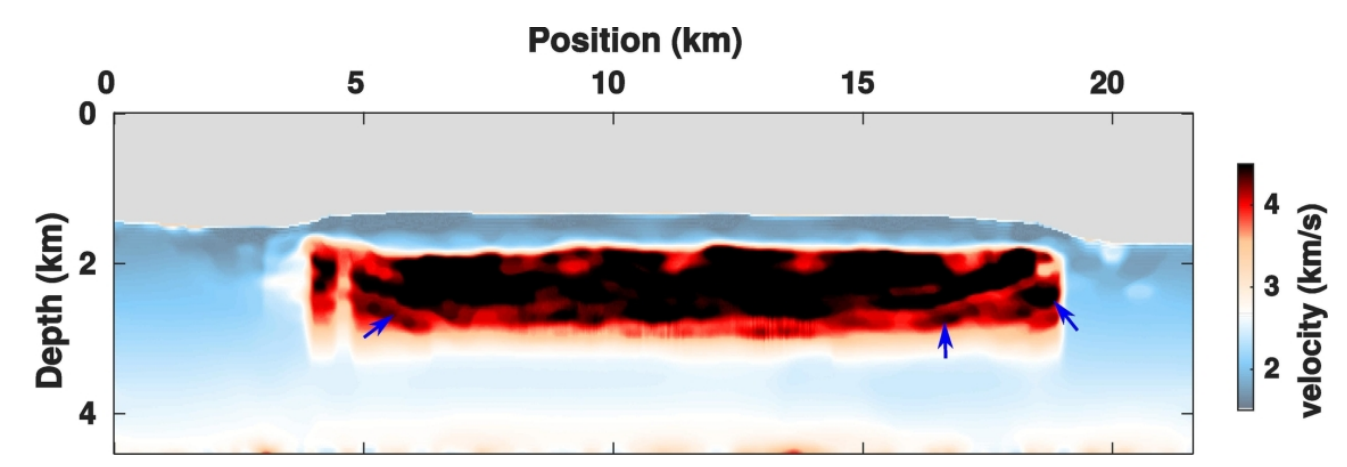

Figure 26: Final inverted model using our proposed methodology. The blue arrows indicate the unexpected over flooding residual.

$178 \times 58 \mathrm{~mm}(300 \times 300 \mathrm{DPI})$ 
Figure 27P1: Shot gather displaying interleaved predicted and observed data using the (a) initial velocity and (b) final velocity model. We interleave 20 traces starting form the predicted followed by observed data. The blue ellipses confirm that the final inverted velocity model predicts a dataset that match the observed ones better than the initial model does.

$239 \times 211 \mathrm{~mm}(300 \times 300 \mathrm{DPI})$ 


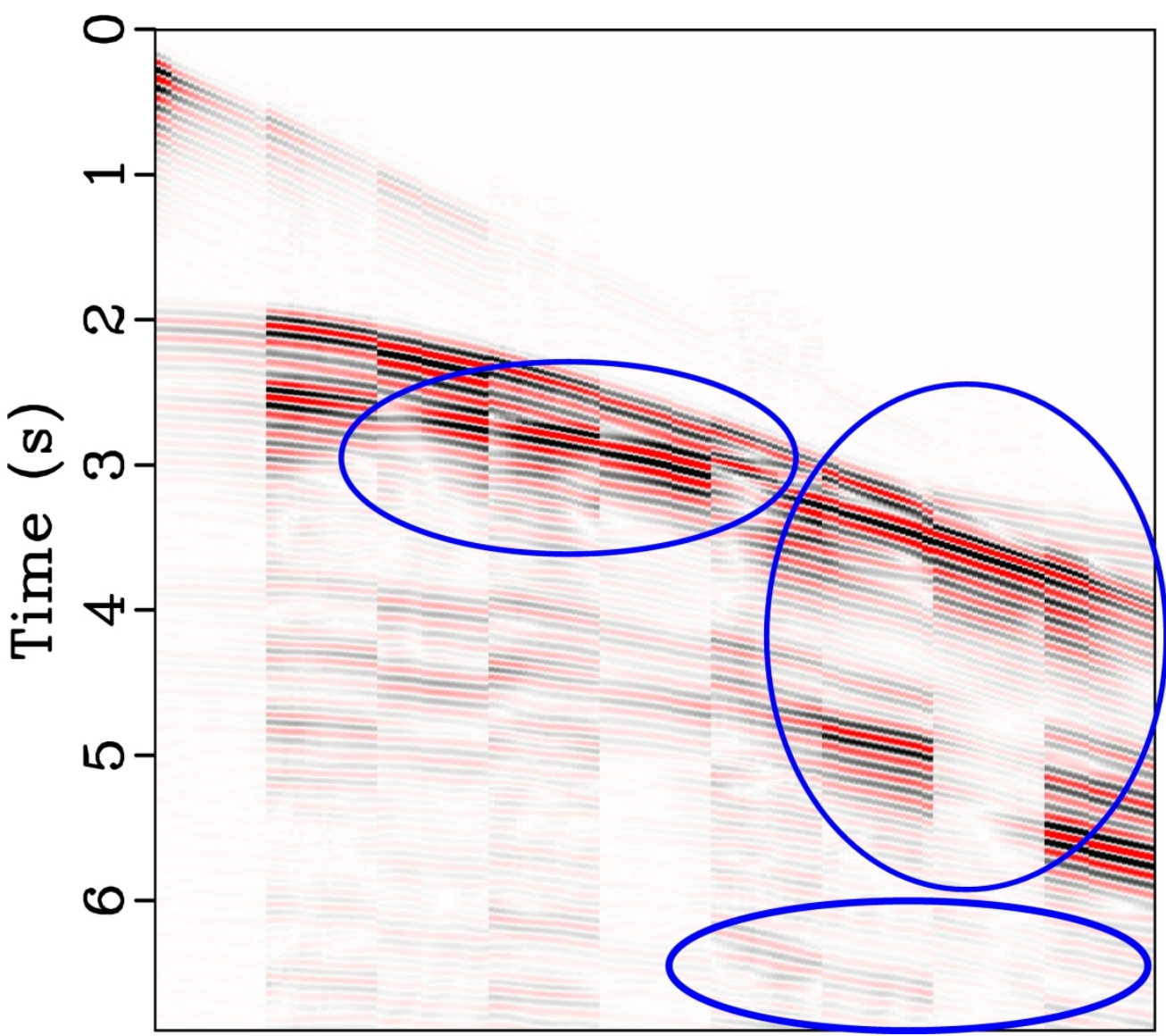

Figure 27P2: Shot gather displaying interleaved predicted and observed data using the (a) initial velocity and (b) final velocity model. We interleave 20 traces starting form the predicted followed by observed data. The blue ellipses confirm that the final inverted velocity model predicts a dataset that match the observed ones better than the initial model does.

$$
241 \times 212 \mathrm{~mm}(300 \times 300 \text { DPI })
$$


Figure 28P1: RTM images using (a) initial velocity and (b) final inverted model. We notice clear boundaries of the salt-dome using the final inverted model, whereas erroneous initial model cannot image those.

$364 \times 114 \mathrm{~mm}(300 \times 300 \mathrm{DPI})$ 
Figure 28P2: RTM images using (a) initial velocity and (b) final inverted model. We notice clear boundaries of the salt-dome using the final inverted model, whereas erroneous initial model cannot image those.

$364 \times 114 \mathrm{~mm}(300 \times 300$ DPI $)$ 


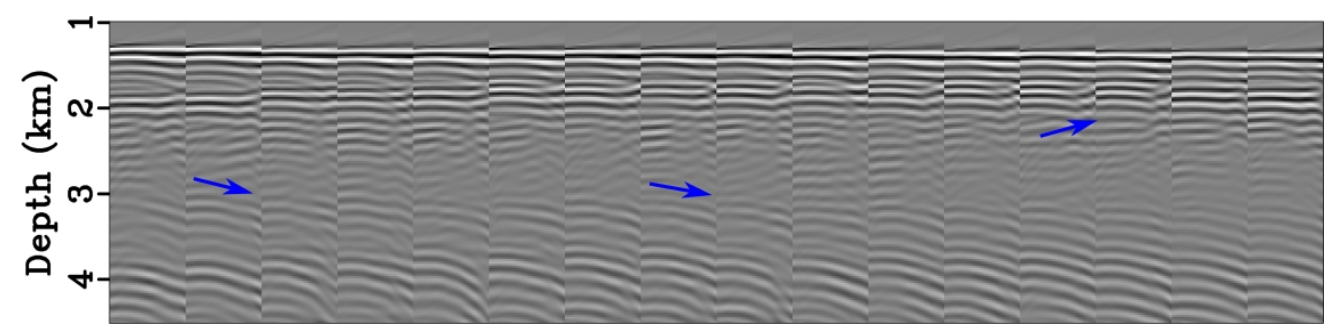

Figure 29P1: ADCIGs $(0 \circ-30 \circ)$ at an interval of $0.7 \mathrm{~km}$, starting from $x=6.0 \mathrm{~km}$ to $x=17.0 \mathrm{~km}$ using (a) initial and (b) final velocity models. We notice events are flatter using the final model as indicated by the arrows.

$327 \times 77 \mathrm{~mm}(300 \times 300 \mathrm{DPI})$ 


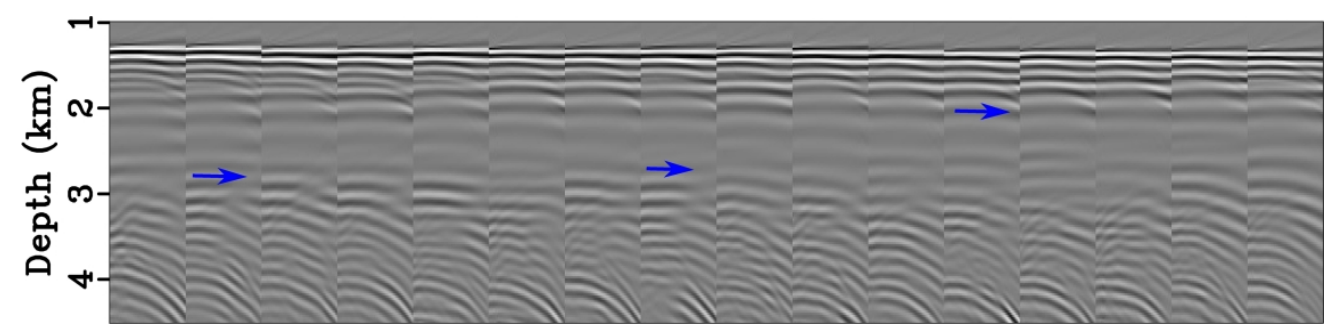

Figure 29: ADCIGs $(0 \circ-30 \circ)$ at an interval of $0.7 \mathrm{~km}$, starting from $x=6.0 \mathrm{~km}$ to $x=17.0 \mathrm{~km}$ using (a) initial and (b) final velocity models. We notice events are flatter using the final model as indicated by the arrows.

$327 \times 77 \mathrm{~mm}(300 \times 300 \mathrm{DPI})$ 
DATA AND MATERIALS AVAILABILITY

Data associated with this research are available and can be obtained by contacting the corresponding author. 